\title{
INTEGRABLE REPRESENTATIONS OF THE QUANTUM AFFINE SPECIAL LINEAR SUPERALGEBRA
}

\author{
YUEZHU WU AND R. B. ZHANG
}

\begin{abstract}
The simple integrable modules with finite dimensional weight spaces are classified for the quantum affine special linear superalgebra $\mathrm{U}_{q}(\widehat{\mathfrak{s l}}(M \mid N))$ at generic $q$. Any such module is shown to be a highest weight or lowest weight module with respect to one of the two natural triangular decompositions of the quantum affine superalgebra depending on whether the level of the module is zero or not. Furthermore, integrable $\mathrm{U}_{q}(\widehat{\mathfrak{s l}}(M \mid N))$-modules at nonzero levels exist only if $M$ or $N$ is 1 .

Key words: quantum supergroups, quantum affine superalgebras, integrable modules, highest weight modules.
\end{abstract}

\section{INTRODUCTION}

Quantum supergroups associated with simple Lie superalgebras and their affine analogues were introduced [2, 30, 45] (see also [4, 11, 27]) in the early 90s, and their structure and representations have since been extensively developed (see, e.g., [1, 16, 17, 19, 21, 28, 32, 33, 35, 38, 41, 42, 46, 47]). Quantum supergroups were applied to solve interesting problems in a variety of areas such as topology of knots and 3-manifolds [13, 36, 39], quantum supergeometry [42, 43], and in particular, YangBaxter type integrable models [2, 10, 44, 32], where the problem of constructing solutions of the spectral parameter dependent Yang-Baxter equation was converted to the much easier linear problem of solving the $\mathbb{Z}_{2}$-graded Jimbo equations [2] by using the representation theory of quantum supergroups.

The $\mathbb{Z}_{2}$-Jimbo equations determine the universal $R$-matrix [16] of quantum affine superalgebras in loop representations. A basic problem in studying the equations is to determine which finite dimensional irreducible representation of a quantum supergroup can be lifted to a representation of the corresponding quantum affine superalgebra. It was shown that the natural representations of quantum orthosymplectic supergroups can be lifted [44], and more importantly, every finite dimensional irreducible representation of the quantum general linear supergroup $\mathrm{U}_{q}(\mathfrak{g l}(M \mid N))$ [38] can be lifted to an irreducible representation of the untwisted quantum affine general linear superalgebra $\mathrm{U}_{q}(\widehat{\mathfrak{g l}}(M \mid N))[35]$.

In a very recent paper [34], Huafeng Zhang gave a classification of the finite dimensional simple modules for $\mathrm{U}_{q}(\widehat{\mathfrak{s l}}(M \mid N))$ (more precisely the subalgebra $\mathrm{U}_{q}^{\prime}(\widehat{\mathfrak{s} l}(M \mid N)$ ) without the degree operator) at generic $q$, providing a parametrisation of such simple 
modules in terms of highest weight polynomials. This has much similarity to the classification [40] of finite dimensional simple modules for the $\mathfrak{g l}(M \mid N)$ super Yangian, as explained in [34].

The present paper generalises results of [25, 26, 29] on $\widehat{\mathfrak{s l}}(M \mid N)$ to the quantum setting to obtain a classification of the simple integrable modules with finite dimensional weight spaces for $\mathrm{U}_{q}(\widehat{\mathfrak{s l}}(M \mid N))$ at generic $q$. A module for a quantum affine superalgebra $\mathrm{U}_{q}(\widehat{\mathfrak{g}})$ is integrable if it is integrable with respect to the subalgebra $\mathrm{U}_{q}\left(\widehat{\mathfrak{g}}_{\overline{0}}\right)$, which is the quantised universal enveloping algebra of the even subalgebra $\widehat{\mathfrak{g}}_{\overline{0}}$ of $\widehat{\mathfrak{g}}$. Thus the integrability of a $\mathrm{U}_{q}(\widehat{\mathfrak{s l}}(M \mid N))$-module amounts to integrability with respect to the subalgebras $\mathrm{U}_{q}(\widehat{\mathfrak{s l}}(M))$ and $\mathrm{U}_{q^{-1}}(\widehat{\mathfrak{s l}}(N))$. The requirement of having finite dimensional weight spaces imposes further stringent conditions on the module.

One result of this paper, Theorem 3.10, states that a zero-level simple integrable module with finite dimensional weight spaces is necessarily of highest weight type with respect to the triangular decomposition of $\mathrm{U}_{q}(\widehat{\mathfrak{s l}}(M \mid N))$ induced by the distinguished triangular decomposition of $\mathfrak{s l}(M \mid N)$ (cf. equation (2.3)). A classification of such modules is given in terms of their highest weight polynomials (see Theorem 3.11).

We show in Theorem 3.11 that any simple integrable $\mathrm{U}_{q}(\widehat{\mathfrak{s l}}(M \mid N))$-module $V$ of zero level with finite dimensional weight spaces can be embedded in a quantum loop module (cf (3.9) ) as a direct summand. By setting the loop parameter to 1, we obtain from the image of $V$ a finite dimensional evaluation $\mathrm{U}_{q}^{\prime}(\widehat{\mathfrak{s l}}(M \mid N))$-module (cf. (3.10) $)$. This way we recover all the finite dimensional simple $\mathrm{U}_{q}^{\prime}(\widehat{\mathfrak{s l}}(M \mid N))$-modules, which were classified in [34].

We prove in Theorem 4.2 that only when $M$ or $N$ is equal to $1, \mathrm{U}_{q}(\widehat{\mathfrak{s l}}(M \mid N))$ admits integrable modules with finite dimensional weight spaces at nonzero levels. Such a simple integrable module is necessarily a highest or lowest weight module with respect to the standard triangular decomposition of $\mathrm{U}_{q}(\widehat{\mathfrak{s l}}(M \mid N))$ given in Proposition 2.2, The necessary and sufficient condition for a simple highest weight $\mathrm{U}_{q}(\widehat{\mathfrak{s l}}(M \mid N))$-module to be integrable with finite dimensional weight spaces is that the highest weight is integral and dominant [8, 18] with respect to the quantised universal enveloping algebra $\mathrm{U}_{q}\left(\widehat{\mathfrak{s l}}(M \mid N)_{\overline{0}}\right)$ of the even subalgebra of $\widehat{\mathfrak{s l}}(M \mid N)$.

We mention that the quantised universal enveloping superalgebras of symmetrizable affine Lie superalgebras (without isotropic odd simple roots) admit many more integrable highest weight modules at nonzero levels. A classification of such simple modules was obtained in [41], where a "super duality" was discovered identifying such quantised universal enveloping superalgebras with certain classes of ordinary quantum affine algebras. 


\section{PRELIMINARIES}

In order to study the integrable modules for the quantum affine special linear superalgebra $\mathrm{U}_{q}(\widehat{\mathfrak{s l}}(M \mid N))$, we need its loop presentation [31], which we discuss here.

2.1. Let us start by discussing some basic structural properties of the special linear superalgebra [14]. Fix positive integers $M$ and $N$, and assume that at least one of them is greater than 1 . Let $I$ be the set $\{1,2, \ldots, M+N-1\}$. We choose the distinguished Borel subalgebra $\mathfrak{b}$ for $\mathfrak{s l}(M \mid N)$, which consists of the upper triangular matrices. The Cartan subalgebra $\mathfrak{h} \subset \mathfrak{b}$ consists of the diagonal matrices in $\mathfrak{s l}(M \mid N)$. Let $\mathfrak{n}$ be the strictly upper triangular matrices, then $\mathfrak{b}=\mathfrak{h} \oplus \mathfrak{n}$.

Equip the free $\mathbb{Z}$-module $\oplus_{i=1}^{M+N} \mathbb{Z} \varepsilon_{i}$ with the following bilinear from

$$
\left(\varepsilon_{i}, \varepsilon_{j}\right)=l_{i} \delta_{i j}, \quad l_{i}= \begin{cases}1, & \text { if } 1 \leq i \leq M \\ -1, & \text { if } M+1 \leq i \leq M+N .\end{cases}
$$

Then the roots of $\mathfrak{s l}(M \mid N)$ can be expressed as $\varepsilon_{i}-\varepsilon_{j}$ for all $i \neq j$, and the simple roots with respect to $\mathfrak{b}$ are given by $\left\{\alpha_{i}:=\varepsilon_{i}-\varepsilon_{i+1} \mid i \in I\right\}$. The even subalgebra $\mathfrak{s l}(M \mid N)_{\overline{0}}$ of $\mathfrak{s l}(M \mid N)$ is $\mathfrak{s l}(M) \oplus \mathbb{C} z \oplus \mathfrak{s l}(N)$, where $\mathbb{C} z$ is the center of $\mathfrak{s l}(M \mid N)_{\overline{0}}$. Let $\mathfrak{h}_{1}$ (resp. $\left.\mathfrak{h}_{2}\right)$ be the Cartan subalgebra of $\mathfrak{s l}(M)$ (resp. $\left.\mathfrak{s l}(N)\right)$, and denote by $\Delta_{0}^{1}$ (resp. $\left.\Delta_{0}^{2}\right)$ the corresponding set of roots. Denote by $Q$ the root lattice of $\mathfrak{s l}(M \mid N)$, and set $Q^{+}=$ $\sum_{i \in I} \mathbb{Z}_{\geq 0} \alpha_{i}$

Let $\widehat{\mathfrak{s l}}(M \mid N)=\mathfrak{s l}(M \mid N) \otimes \mathbb{C}\left[t, t^{-1}\right] \oplus \mathbb{C} c \oplus \mathbb{C} d$ be the untwisted extended affine Lie superalgebra associated with $\mathfrak{s l}(M \mid N)$, where $c$ spans the center, and $d$ is the degree operator. We take the following Cartan subalgebra $\widehat{\mathfrak{h}}=\mathfrak{h} \otimes 1 \oplus \mathbb{C} c \oplus \mathbb{C} d$ for $\widehat{\mathfrak{s l}}(M \mid N)$. Introduce the affine weight $\omega_{0}$ and null root $\delta$ in $\mathfrak{h}^{*}$ such that $\omega_{0}(c)=1, \delta(d)=1$, and $\omega_{0}(h)=\delta(h)=0, \forall h \in \mathfrak{h}$. Then

$$
\left(\omega_{0}, \alpha_{i}\right)=\left(\delta, \alpha_{i}\right)=0, \forall i \in I, \quad\left(\omega_{0}, \omega_{0}\right)=(\delta, \delta)=0, \quad\left(\omega_{0}, \delta\right)=1 .
$$

Then $\omega_{0}, \delta$ and all the $\alpha_{i}$ together form a basis of $\mathfrak{h}^{*}$. Denote by $\widehat{Q}$ the $\mathbb{Z}$-span of the $\alpha_{i}$ and $\delta$, i.e., the root lattice of $\widehat{\mathfrak{s l}}(M \mid N)$, and let $\alpha_{0}=\delta-\sum_{i \in I} \alpha_{i}$.

Recall that we have the following Borel subalgebras of $\widehat{\mathfrak{s l}}(M \mid N)$,

$$
\begin{aligned}
& \mathbb{C} c \oplus \mathbb{C} d \oplus \mathfrak{b} \oplus \mathfrak{s l}(M \mid N) \otimes t \mathbb{C}[t], \\
& \mathbb{C} c \oplus \mathbb{C} d \oplus \mathfrak{b} \otimes \mathbb{C}\left[t, t^{-1}\right],
\end{aligned}
$$

where (2.1) is the standard Borel subalgebra, while (2.2) is induced by $\mathfrak{b} \subset \mathfrak{s l}(M \mid N)$. Later we will make use of quantum universal enveloping superalgebras of these Borel subalgebras.

2.2. Let us fix once for all a nonzero complex number $q$ which is not a root of 1 . For any $m \in \mathbb{Z}_{+}$, define $[m]_{q}=\frac{q^{m}-q^{-m}}{q-q^{-1}}$. Let $q_{i}=q^{\left(\varepsilon_{i}, \varepsilon_{i}\right)}$ for $i \in I$, and set

$$
a_{i j}=\left(\varepsilon_{i}-\varepsilon_{i+1}, \varepsilon_{j}-\varepsilon_{j+1}\right), \quad \text { for all } i, j \in I \text {. }
$$


The quantum affine superalgebra $\widehat{\mathrm{U}}_{q}:=\mathrm{U}_{q}(\widehat{\mathfrak{s l}}(M \mid N))$ is a Hopf superalgebra over $\mathbb{C}[2,[16,31,35,45]$, which has two presentations, a Serre presentation in terms of Chevalley generators and Serre type relations, and loop presentation. Its loop presentation was constructed in [31].

Definition 2.1. The loop presentation of $\widehat{\mathrm{U}}_{q}:=\mathrm{U}_{q}(\widehat{\mathfrak{s l}}(M \mid N))$ is as follows. The set of generators is

$$
\left\{X_{i}^{ \pm}(n), K_{i}^{ \pm 1}, h_{i}(s), C^{ \pm 1 / 2}, D^{ \pm 1} \mid i \in I, n, s \in \mathbb{Z}, s \neq 0\right\},
$$

where $X_{M}^{ \pm}(m)$ for all $m \in \mathbb{Z}$ are odd, and the other elements are even.

The relations are

$$
\begin{aligned}
& C^{ \pm 1 / 2} \text { are central, } \\
& K_{i} K_{i}^{-1}=1=K_{i}^{-1} K_{i}, \quad\left[K_{i}, K_{j}\right]=\left[K_{i}, h_{j}(s)\right]=0, \quad\left[K_{i}, D\right]=0, \\
& D D^{-1}=D^{-1} D=1, \quad D h_{i}(s) D^{-1}=q^{s} h_{i}(s), \quad D X_{i}^{ \pm}(s) D^{-1}=q^{s} X_{i}^{ \pm}(s) \text {, } \\
& K_{i} X_{j}^{ \pm}(n) K_{i}^{-1}=q^{ \pm a_{i j}} X_{j}^{ \pm}(n) \text {, } \\
& {\left[h_{i}(m), h_{j}(n)\right]=\delta_{m+n, 0} \frac{\left[m l_{i} a_{i j}\right]_{q_{i}}\left(C^{m}-C^{-m}\right)}{m\left(q_{i}-q_{i}^{-1}\right)},} \\
& {\left[h_{i}(s), X_{j}^{ \pm}(n)\right]= \pm \frac{\left[s l_{i} a_{i j}\right] q_{i}}{s} C^{\mp \frac{|s|}{2}} X_{j}^{ \pm}(n+s),} \\
& {\left[X_{i}^{+}(m), X_{j}^{-}(n)\right]=\delta_{i j} \frac{C^{(m-n) / 2} \phi_{i}^{+}(m+n)-C^{-(m-n) / 2} \phi_{i}^{-}(m+n)}{q_{i}-q_{i}^{-1}},} \\
& {\left[X_{i}^{ \pm}(m), X_{j}^{ \pm}(n)\right]=0 \quad \text { for } a_{i j}=0,} \\
& {\left[X_{i}^{ \pm}(m+1), X_{j}^{ \pm}(n)\right]_{q^{ \pm a_{i j}}}+\left[X_{j}^{ \pm}(n+1), X_{i}^{ \pm}(m)\right]_{q^{ \pm a_{i j}}}=0 \quad \text { for } a_{i j} \neq 0,}
\end{aligned}
$$

and

$$
\begin{aligned}
& \operatorname{Sym}_{m, n}\left[X_{i}^{ \pm}(m),\left[X_{i}^{ \pm}(n), X_{j}^{ \pm}(k)\right]_{q^{-1}}\right]_{q}=0 \quad \text { for } a_{i j}= \pm 1, i \neq M, \\
& \operatorname{Sym}_{n, u}\left[\left[\left[X_{M-1}^{ \pm}(m), X_{M}^{ \pm}(n)\right]_{q^{-1}}, X_{M+1}^{ \pm}(k)\right]_{q}, X_{M}^{ \pm}(u)\right]=0, \quad \text { when } M, N>1,
\end{aligned}
$$

where $\phi_{i}^{ \pm}(n)$ are given by the generating series

$$
\sum_{n \in \mathbb{Z}} \phi_{i}^{ \pm}(n) z^{n}=K_{i}^{ \pm 1} \exp \left(\left(q_{i}-q_{i}^{-1}\right) \sum_{s \in \mathbb{Z}_{>0}} h_{i}( \pm s) z^{ \pm s}\right) \in \widehat{\mathrm{U}}_{q}\left[\left[z, z^{-1}\right]\right],
$$

and the symbol $\mathrm{Sym}_{k, l}$ means symmetrization with respect to $k$ and $l$. We have used the notation of $q$-brackets $[X, Y]_{u}=X Y-(-1)^{|X||Y|} u Y X$, and written $[X, Y]$ for $[X, Y]_{1}$ for simplicity.

We denote by $\mathrm{U}_{q}^{\prime}(\widehat{\mathfrak{s l}}(M \mid N))$ the subalgebra of $\widehat{\mathrm{U}}_{q}$ without the generators $D^{ \pm 1}$. By dropping the generators $X_{M}^{ \pm}(n)$ for all $n \in \mathbb{Z}$, we obtain a subalgebra of $\widehat{\mathrm{U}}_{q}$, which is the quantised universal enveloping algebra $\mathrm{U}_{q}\left(\widehat{\mathfrak{s l}}(M \mid N)_{\overline{0}}\right)$ of the even subalgebra 
$\mathfrak{s l}(M \mid N)_{\overline{0}}$ of $\mathfrak{s l}(M \mid N)$. Note that this subalgebra contains $\mathrm{U}_{q}(\widehat{\mathfrak{s l}}(M))$ and $\mathrm{U}_{q^{-1}}(\widehat{\mathfrak{s l}}(N))$ as subalgebras.

The superalgebra $\widehat{\mathrm{U}}_{q}$ is $\mathbb{Z}$-graded $\widehat{\mathrm{U}}_{q}=\oplus_{k \in \mathbb{Z}}\left(\widehat{\mathrm{U}}_{q}\right)_{k}$ with homogeneous components $\left(\widehat{\mathrm{U}}_{q}\right)_{k}=\left\{x \in \widehat{\mathrm{U}}_{q} \mid D x D^{-1}=q^{k} x\right\}$. Let us introduce the following subalgebras of $\widehat{\mathrm{U}}_{q}$ :

- $\widehat{\mathrm{U}}_{q}^{+}(\gg)$ (resp. $\widehat{\mathrm{U}}_{q}^{+}(\ll)$ ) denotes the subalgebra generated by the elements $X_{i}^{+}(m)$ for all $m \geq 0$ and $i \in I$ (resp. $X_{i}^{+}(m)$ for all $m<0$ and $i \in I$ );

- $\widehat{\mathrm{U}}_{q}^{-}(\gg)$ (resp. $\widehat{\mathrm{U}}_{q}^{-}(\ll)$ ) denotes the subalgebra generated by the elements $X_{i}^{-}(m)$ for all $m>0$ and $i \in I$ (resp. $X_{i}^{-}(m)$ for all $m \leq 0$ and $i \in I$ );

- $\widehat{\mathrm{U}}_{q}^{0}(\gg)$ (resp. $\widehat{\mathrm{U}}_{q}^{0}(\ll)$ ) denotes the subalgebra generated by the elements $h_{i}(r)$ for all $r>0$ and $i \in I$ (resp. $h_{i}(r)$ for all $r<0$ and $i \in I$ );

- $\widehat{\mathrm{U}}_{q}^{0}$ denotes the subalgebra generated by $K_{i}^{ \pm 1}(i \in I), D^{ \pm 1}$ and $C^{ \pm 1 / 2}$.

We have the following obvious result.

Proposition 2.2. Define the following subspaces of $\widehat{\mathrm{U}}_{q}$

$$
\widehat{\mathrm{U}}_{q}(+):=\widehat{\mathrm{U}}_{q}^{+}(\gg) \widehat{\mathrm{U}}_{q}^{-}(\gg) \widehat{\mathrm{U}}_{q}^{0}(\gg), \quad \widehat{\mathrm{U}}_{q}(-):=\widehat{\mathrm{U}}_{q}^{+}(\ll) \widehat{\mathrm{U}}_{q}^{-}(\ll) \widehat{\mathrm{U}}_{q}^{0}(\ll) .
$$

Then $\widehat{\bar{B}}=\widehat{\mathrm{U}}_{q}(-) \widehat{\mathrm{U}}_{q}(0)$ and $\widehat{B}=\widehat{\mathrm{U}}_{q}(0) \widehat{\mathrm{U}}_{q}(+)$ are subalgebras, and

$$
\widehat{\mathrm{U}}_{q}=\widehat{\mathrm{U}}_{q}(-) \widehat{\mathrm{U}}_{q}^{0} \widehat{\mathrm{U}}_{q}(+) \text {. }
$$

Note that $\widehat{B}$ is the quantised universal enveloping algebra of the Borel subalgebra of $\widehat{\mathfrak{s l}}(M \mid N)$ given in (2.1). Thus this triangular decomposition of $\widehat{\mathrm{U}}_{q}$ is the quantum analogue of the triangular decomposition of $\mathrm{U}(\widehat{\mathfrak{s l}}(M \mid N))$ with respect to the Borel subalgebra (2.1).

2.3. Let $\mathrm{U}_{q}$ be the extended quantum loop superalgebra, namely the quotient of $\widehat{\mathrm{U}}_{q}$ by the ideal generated by $C^{ \pm 1 / 2}-1$, and denote by $\mathrm{U}_{q}^{\prime}$ the $\mathbb{C}$-subalgebra of $\mathrm{U}_{q}$ without the generators $D^{ \pm 1}$. Define the following subalgebras of $\mathrm{U}_{q}$ :

- $\mathrm{U}_{q}(0)$ denotes the subalgebra generated by $h_{i}(r), K_{i}^{ \pm 1}, D^{ \pm 1}$ for all $i \in I$ and $0 \neq r \in \mathbb{Z}$;

- $\mathrm{U}_{q}^{+}$(resp. $\mathrm{U}_{q}^{-}$) denotes the subalgebra generated by $X_{i}^{+}(m)$ for all $i \in I$ and $m \in \mathbb{Z}$ (resp. $X_{i}^{-}(m)$ for all $i \in I$ and $m \in \mathbb{Z}$ ),

and let $\mathrm{U}_{q}^{\prime}(0)=\mathrm{U}_{q}(0) \cap \mathrm{U}_{q}^{\prime}$, which is a subalgebra of $\mathrm{U}_{q}^{\prime}$. Then

$$
\mathrm{U}_{q}=\mathrm{U}_{q}^{-} \mathrm{U}_{q}(0) \mathrm{U}_{q}^{+}, \quad \mathrm{U}_{q}^{\prime}=\mathrm{U}_{q}^{-} \mathrm{U}_{q}^{\prime}(0) \mathrm{U}_{q}^{+} .
$$

Define the following subalgebras of $\mathrm{U}_{q}$ and $\mathrm{U}_{q}^{\prime}$ respectively:

$$
B:=\mathrm{U}_{q}(0) \mathrm{U}_{q}^{+}, \quad B^{\prime}:=\mathrm{U}_{q}^{\prime}(0) \mathrm{U}_{q}^{+} .
$$

Then $B$ can be considered as the quantised universal enveloping superalgebra of the Borel subalgebra of $\widehat{\mathfrak{s l}}(M \mid N)$ given in (2.2) without the central element, and $B^{\prime}$ is the 
subalgebra of $B$ without the generators $D^{ \pm 1}$. Thus the triangular decompositions (2.3) are quantum analogues of the triangular decomposition of $\mathrm{U}(\widehat{\mathfrak{s l}}(M \mid N))$ with respect to the Borel subalgebra (2.2) of $\widehat{\mathfrak{s l}}(M \mid N)$.

Set $\Delta=\left\{\beta_{i j}:=\alpha_{i}+\alpha_{i+1}+\cdots+\alpha_{j} \mid i, j \in I, i \leq j\right\}$ with the following total ordering $\beta_{i, j}<\beta_{i^{\prime}, j^{\prime}}$ if $i<i^{\prime}$ or $i=i^{\prime}, j<j^{\prime}$. For $\beta_{i, j} \in \Delta$ and $n \in \mathbb{Z}$, define

$$
X_{i, j}^{+}(n)=\left[\cdots\left[\left[X_{i}^{+}(n), X_{i+1}^{+}(0)\right]_{q_{i+1}}, X_{i+2}^{+}(0)\right]_{q_{i+2}}, \cdots, X_{j}^{+}(0)\right]_{q_{j}},
$$

with the convention that $X_{i, i}^{+}(n)=X_{i}^{+}(n)$.

Proposition 2.3. [34, Theorem 3.12] $\mathrm{U}_{q}^{+}$is spanned by vectors of the form

$$
\prod_{1 \leq a \leq b \leq M+N-1}^{\rightarrow}\left(\prod_{i=1}^{c_{a b}} X_{a, b}^{+}\left(n_{a b, i}\right)\right), \quad c_{a b} \in \mathbb{Z}_{\geq 1}, n_{a b, i} \in \mathbb{Z}
$$

where $\vec{\prod}$ is the ordered product positioning $X_{a, b}^{+}(m)$ on the left of $X_{a^{\prime}, b^{\prime}}^{+}(n)$ if $\beta_{a, b}<$ $\beta_{a^{\prime}, b^{\prime}}$.

2.4. All the modules for $\widehat{U}_{q}$ and $\widehat{U}_{q}^{\prime}$ considered in this paper are assumed to be $\mathbb{Z}_{2}$ graded. Given a $\widehat{U}_{q}$-module $V$, let

$$
V_{\mu}=\left\{v \in V \mid D v=q^{\left(\mu, \omega_{0}\right)} v, C^{ \pm 1 / 2} v=q^{ \pm \frac{1}{2}(\mu, \delta)} v, K_{i} v=q_{i}^{\left(\mu, \alpha_{i}\right)} v, i \in I\right\}
$$

for any $\mu \in \widehat{\mathfrak{h}}^{*}$. If $V_{\mu} \neq 0$, we say that $\mu$ is a weight of $V$, and denote by $P(V)$ the set of the weights. The module $V$ is said to be a weight module of type 1 if

$$
V=\bigoplus_{\mu \in P(V)} V_{\mu}
$$

From now on, all modules will be assumed to be of type 1. A $\widehat{\mathrm{U}}_{q}$ module $V$ is integrable if $V=\bigoplus_{\mu \in P(V)} V_{\mu}$, and the elements $X_{i}^{ \pm}(m)(i \in I, m \in \mathbb{Z})$ act locally nilpotently. If $C^{ \pm 1 / 2}$ act by the identity, we say that $V$ is a zero-level module, or at level 0.

\section{ZERO-LEVEL INTEGRABLE REPRESENTATIONS FOR $\widehat{\mathrm{U}}_{q}$}

In this section we classify the irreducible integrable $\widehat{U}_{q}$-modules with finite dimensional weight spaces such that $C^{ \pm 1 / 2}$ act as the identity. Such modules descend to $\mathrm{U}_{q}$-modules.

3.1. Let $H$ (resp. $H^{\prime}$ ) be the subalgebra of $\mathrm{U}_{q}$ generated by $K_{i}^{ \pm 1}, D^{ \pm 1}, i \in I$ (resp. $K_{i}^{ \pm 1}, i \in I$ ). A module $V$ of $\mathrm{U}_{q}$ (resp. $\mathrm{U}_{q}^{\prime}$ ) is called a highest weight module if there exists a nonzero weight vector $v \in V$ with respect to $H$ (resp. $H^{\prime}$ ) such that

(1) $\mathrm{U}_{q} v=V$ (resp. $\mathrm{U}_{q}^{\prime} v=V$ ),

(2) $X_{i}^{+}(m) v=0$ for all $i \in I$ and $m \in \mathbb{Z}$, and

(3) $\mathrm{U}_{q}(0) v\left(\right.$ resp. $\left.\mathrm{U}_{q}^{\prime}(0) v\right)$ is an irreducible $\mathrm{U}_{q}(0)$-module (resp. $\mathrm{U}_{q}^{\prime}(0)$-module). 
Call $v$ a highest weight vector of $V$ relative to $B\left(\right.$ resp. $\left.B^{\prime}\right)$ as $B v=\mathbb{C} v\left(\right.$ resp. $\left.B^{\prime} v=\mathbb{C} v\right)$. These highest weight modules are defined relative to the triangular decompositions for $\mathrm{U}_{q}$ (resp. $\mathrm{U}_{q}^{\prime}$ ) defined by (2.3).

Let $\psi: \mathrm{U}_{q}^{\prime}(0) \rightarrow \mathbb{C}$ be any algebra homomorphism, and let $\mathrm{U}_{q}^{\prime}(0)$ act on the one dimensional vector space $\mathbb{C}_{\psi}=\mathbb{C}$ by $\psi$. We extend $\mathbb{C}_{\psi}$ to a module over $B^{\prime}$ (cf. (2.4) ) by letting $\mathrm{U}_{q}^{+}$act trivially. Construct the induced $\mathrm{U}_{q}^{\prime}$-module

$$
M(\psi)=\mathrm{U}_{q}^{\prime} \otimes_{B^{\prime}} \mathbb{C}_{\psi}
$$

which has a unique simple quotient:

$$
V(\psi)=\text { the simple quotient } \mathrm{U}_{q}^{\prime} \text {-module of } M(\psi) .
$$

The following definition is taken from [34].

Definition 3.1. [34] Let $\mathcal{R}_{M, N}$ be the set consisting of elements $(P, f, c, Q)$, where

(1) $f(z)=\sum_{n \in \mathbb{Z}} f_{n} z^{n} \in \mathbb{C}\left[\left[z, z^{-1}\right]\right]$ is a formal series and $Q(z) \in 1+z \mathbb{C}[z]$ is a polynomial such that

$$
Q(z) f(z)=0
$$

(2) $c \in \mathbb{C} \backslash\{0\}$ with $\frac{c-c^{-1}}{q-q^{-1}}=f_{0}$;

(3) $P=\left(P_{1}, \ldots, P_{M-1}, P_{M+1}, \ldots, P_{M+N-1}\right)$ with $P_{i} \in 1+z \mathbb{C}[z]$.

With the help of results from [34], we can characterise the integrability of $V(\psi)$ as follows.

Theorem 3.2. The following are equivalent for the simple $\mathrm{U}_{q}^{\prime}$-module $V(\psi)(c f$. (3.1)).

(1) $V(\psi)$ is an integrable $\mathrm{U}_{q}^{\prime}$-module with finite dimensional weight spaces.

(2) There exists $(P, f, c, Q) \in \mathcal{R}_{M, N}$ such that for any highest weight vector $v \in$ $V(\psi)$

$$
\begin{aligned}
& X_{i}^{+}(n) v=0 \quad \text { for } i \in I, n \in \mathbb{Z}, \\
& \psi\left(\sum_{n \in \mathbb{Z}} \phi_{i}^{ \pm}(n) z^{n}\right) v=q_{i}^{\operatorname{deg} P_{i}} \frac{P_{i}\left(z q_{i}^{-1}\right)}{P_{i}\left(z q_{i}\right)} v \in \mathbb{C}\left[\left[z^{ \pm 1}\right]\right], \quad i \in I, i \neq M, \\
& \left(X_{i}^{-}(0)\right)^{1+\operatorname{deg} P_{i}} v=0, \quad i \in I, i \neq M, \\
& \psi\left(K_{M}\right) v=c v, \quad \psi\left(\sum_{n \in \mathbb{Z}} \frac{\phi_{M}^{+}(n)-\phi_{M}^{-}(n)}{q-q^{-1}} z^{n}\right) v=f(z) v, \\
& \sum_{s=0}^{d} a_{d-s} X_{M}^{-}(s+r) v=0, \quad \forall r \in \mathbb{Z}, \text { with } Q(z)=\sum_{s=0}^{d} a_{s} z^{s},
\end{aligned}
$$

where (3.3) is understood as an equation of Laurent series expanded about $z=0$ for $\phi_{i}^{+}$(resp. $z=\infty$ for $\phi_{i}^{-}$).

(3) $V(\psi)$ is finite dimensional. 
Proof. (1) $\Rightarrow(2)$. When $i \neq M$, let $\mathrm{U}_{q}^{(i)}$ be the $\mathrm{U}_{q_{i}}(\widehat{\mathfrak{s l}}(2))$ subalgebra generated by

$$
X_{i}^{ \pm}(n), K_{i}^{ \pm 1}, h_{i}(r), \quad n, r \in \mathbb{Z}, r \neq 0 .
$$

Then $\mathrm{U}_{q}^{(i)} v$ is an integrable highest weight $\mathrm{U}_{q}^{(i)}$-module. By [8, Theorem 3.4], there exists a polynomial $P_{i} \in 1+z \mathbb{C}[z]$ satisfying (3.3) and (3.4).

When $i=M$, there exist $c \in \mathbb{C} \backslash\{0\}, f_{n} \in \mathbb{C}, n \in \mathbb{Z}$ such that

$$
\begin{aligned}
& K_{M} v=c v, \\
& \frac{\phi_{M}^{+}(n)-\phi_{M}^{-}(n)}{q-q^{-1}} v=\psi\left(\frac{\phi_{M}^{+}(n)-\phi_{M}^{-}(n)}{q-q^{-1}}\right) v=f_{n} v, \quad n \neq 0, \\
& \frac{\phi_{M}^{+}(0)-\phi_{M}^{-}(0)}{q-q^{-1}} v=\psi\left(\frac{K_{M}-K_{M}^{-1}}{q-q^{-1}}\right) v=f_{0} v .
\end{aligned}
$$

Since $X_{M}^{-}(n) v$ for all $n \in \mathbb{Z}$ belong to the same weight space of $V(\psi)$, and all weight spaces are finite dimensional, there exist $m \in \mathbb{Z}, d \in \mathbb{Z}_{\geq 0}$ and $a_{0}, \ldots, a_{d} \in \mathbb{C}$ satisfying $a_{d} \neq 0$ and $a_{0}=1$ such that

$$
\sum_{s=0}^{d} a_{d-s} X_{M}^{-}(s+m) v=0 .
$$

Applying $h_{M-1}(r)$ to the above equality, we obtain

$$
\begin{aligned}
0 & =\sum_{s=0}^{d} a_{d-s}\left[h_{M-1}(r), X_{M}^{-}(s+m)\right] v+\sum_{s=0}^{d} a_{d-s} X_{M}^{-}(s+m) h_{M-1}(r) v \\
& =\sum_{s=0}^{d} a_{d-s} \frac{[r]_{q}}{r} X_{M}^{-}(s+m+r) v+\sum_{s=0}^{d} a_{d-s} X_{M}^{-}(s+m) \psi\left(h_{M-1}(r)\right) v \\
& =\frac{[r]_{q}}{r}\left(\sum_{s=0}^{d} a_{d-s} X_{M}^{-}(s+m+r) v\right) .
\end{aligned}
$$

Hence, $\sum_{s=0}^{d} a_{d-s} X_{M}^{-}(s+m+r) v=0$ and (3.6) holds.

Applying $X_{M}^{+}(0)$ to $\sum_{s=0}^{d} a_{d-s} X_{M}^{-}(s+m+r) v=0$, we have

$$
\begin{aligned}
X_{M}^{+}(0) \sum_{s=0}^{d} a_{d-s} X_{M}^{-}(s+m+r) v & =\sum_{s=0}^{d} a_{d-s} \frac{\phi_{M}^{+}(s+m+r)-\phi_{M}^{-}(s+m+r)}{q-q^{-1}} v \\
& =\sum_{s=0}^{d} a_{d-s} f_{s+m+r} v=0
\end{aligned}
$$

which implies that $Q(z)\left(\sum_{n \in \mathbb{Z}} f_{n} z^{n}\right)=0$, where $Q(z)=\sum_{s=0}^{d} a_{s} z^{s}$. 
$(2) \Rightarrow(3)$. This was established in [34, Theorem 4.5], which is a key result in the classification of finite dimensional simple $\mathrm{U}_{q}^{\prime}$-modules.

(3) $\Rightarrow(1)$. Clear.

Definition 3.3. We will denote by $V(P, f, c, Q)$ the $\mathrm{U}_{q}^{\prime}$-module $V(\psi)$ corresponding to $(P, f, c, Q) \in \mathcal{R}_{M, N}$ in Theorem 3.2 and call $P, f$ and $Q$ the highest weigh polynomials of $V(P, f, c, Q)$.

Note that $f$ is a formal Laurent series in general.

3.2. The following result is [6, Lemma 1.4].

Lemma 3.4. Let $\chi: \mathrm{U}_{q}^{\prime}(0) \rightarrow \mathbb{C}\left[t, t^{-1}\right]$ be a nontrivial homomorphism of $\mathbb{Z}$-graded algebras. Then there exists a unique $r>0$ such that the image of $\chi$ equals to $\mathbb{C}\left[t^{r}, t^{-r}\right]$.

Let $\tilde{\varphi}: \mathrm{U}_{q}^{\prime}(0) \rightarrow L:=\mathbb{C}\left[t, t^{-1}\right]$ be a $\mathbb{Z}$-graded algebra homomorphism such that $\tilde{\varphi}\left(C^{ \pm 1 / 2}\right)=1$ and $\tilde{\varphi}\left(K_{i}^{ \pm 1}\right) \in \mathbb{C} \backslash\{0\}$. Then for any given $b \in \mathbb{C}$, we can turn $L$ into a $\mathrm{U}_{q}(0)$-module via $\tilde{\varphi}$ defined for all $f \in L$ by

$$
D f=q^{t \frac{d}{d t}+b} f, \quad x f=\tilde{\varphi}(x) f, \quad x \in \mathrm{U}_{q}^{\prime}(0) .
$$

We write $\varphi=(\tilde{\varphi}, b)$ and denote by $L_{\varphi}$ the image of $\tilde{\varphi}$ regarded as a $\mathbb{Z}$-graded $\mathrm{U}_{q}(0)$ submodule. Then $L_{\varphi}$ is $L_{0}:=\mathbb{C}$ or a Laurent subring $L_{r}:=\mathbb{C}\left[t^{r}, t^{-r}\right]$ for some integer $r>0$. This follows from Lemma 3.4 .

Assume that $L_{\varphi}$ is a simple $\mathrm{U}_{q}(0)$-module. We extend $L_{\varphi}$ to a module over $B$ (cf. (2.4)) with $\mathrm{U}_{q}^{+}$acting trivially, and construct the induced $\mathrm{U}_{q}$-module

$$
M(\varphi)=\mathrm{U}_{q} \otimes_{B} L_{\varphi}
$$

This has a unique irreducible quotient, which we denote by $V(\varphi)$. Then every irreducible highest weight $\mathrm{U}_{q}$-module is isomorphic to some $V(\varphi)$.

Given any simple $\mathrm{U}_{q}^{\prime}$-module $V(\psi)$ (cf. (3.1)), we form the vector space $V(\psi) \otimes L$ and denote $w(s)=w \otimes t^{s}$ for any $w \in V(\psi)$ and $s \in \mathbb{Z}$. For any $b \in \mathbb{C}$, we now turn $V(\psi) \otimes L$ into a $\mathrm{U}_{q}$-module by defining the action

$$
\begin{aligned}
& C^{ \pm 1 / 2} w(s)=w(s), \quad D w(s)=q^{s+b} w(s), \\
& x w(s)=(x w)(s+m), \quad x \in\left(\mathrm{U}_{q}\right)_{m} .
\end{aligned}
$$

We denote this $\mathrm{U}_{q}$-module by $L(V(\psi) ; b)$ and call it the quantum loop module associated to $V(\psi)$ and $b$. Then $V(\psi)$ is an integrable $\mathrm{U}_{q}^{\prime}$-module if and only if $L(V(\psi) ; b)$ is an integrable $\mathrm{U}_{q}$-module.

Theorem 3.5. Let $V(\varphi)$ be a $\widehat{\mathrm{U}}_{q}$-module such that $L_{\varphi} \cong L_{r}$ is an irreducible $\mathrm{U}_{q}^{\prime}(0)$ module. Define $\psi=S \circ \tilde{\varphi}: \mathrm{U}_{q}^{\prime}(0) \rightarrow \mathbb{C}$ with $S: L \rightarrow \mathbb{C}, t \mapsto 1$, being the evaluation map. Let $v$ be a highest weight vector of $V(\psi)$ and denote $v(i)=v \otimes t^{i}$ for any $i \in \mathbb{Z}$. Then 
(1) $V(\psi) \otimes L \cong \oplus_{i=0}^{r-1} \mathrm{U}_{q} v(i)$ as $\mathrm{U}_{q}$-modules, where $\mathrm{U}_{q}$-submodules $\mathrm{U}_{q} v(i)$ are simple. Furthermore, $\mathrm{U}_{q} v(0) \cong V(\varphi)$.

(2) $V(\varphi)$ has finite dimensional weight spaces with respect to $H$ if and only if $V(\psi)$ has finite dimensional weight spaces with respect to $H^{\prime}$.

Proof. The proofs of [22, Theorem 1.8] and [23, Lemma 1.10] can be adopted verbatim to prove this result. We refer the interested readers to the paper [22, 23] for details.

We note that $\mathrm{U}_{q} v(i) \cong V(\tilde{\varphi}, b+i)$. In the case $r=0$, the formula in part (1) of the theorem should be understood as $V(\psi) \otimes L \cong \oplus_{i \in \mathbb{Z}} \mathrm{U}_{q} v(i)$.

Given any nonzero simple $\mathrm{U}_{q}$-submodule $\mathrm{U}_{q} v(i) \subseteq L(V(\psi), b)$, we define the evaluation module for $\mathrm{U}_{q}^{\prime}$ by setting $t=1$ :

$$
\mathrm{U}_{q} v(i) \longrightarrow V(\psi), \quad w(s) \mapsto w .
$$

This is a $\mathrm{U}_{q}^{\prime}$-module homomorphism, which is surjective.

3.3. Let $V$ be an irreducible integrable $\mathrm{U}_{q}$-module with finite dimensional weight spaces. In this section we generalize the method developed in [29] to show that $V$ has to be a highest weight module with respect to the triangular decomposition of $\mathrm{U}_{q}$ given in (2.3).

Introduce the set $S=\{(a, b) \mid 1 \leq a \leq M \leq b \leq M+N-1, a<b\}$, and order the elements so that $(a, b)>\left(a^{\prime}, b^{\prime}\right)$ if and only if $b-a>b^{\prime}-a^{\prime}$ or $b-a=b^{\prime}-a^{\prime}, a<a^{\prime}$.

We have the following lemmas, which play a key role in the remainder of the section. Their proofs are relegated to Appendices $[\mathrm{B}$ and $\mathrm{C}$ as they involve very lengthy computations.

Lemma 3.6. Let $v_{a-1, b-1}$ be a weight vector in $V$. For any $n_{1}, \ldots, n_{p} \in \mathbb{Z}$ and $p \in \mathbb{Z}_{>0}$, denote

$$
\begin{gathered}
v_{a, b}:=X_{a, b}^{+}\left(n_{p}\right) \cdots X_{a, b}^{+}\left(n_{1}\right) v_{a-1, b-1}, \text { if } b-1 \neq M+N, \\
\left(\text { resp. } v_{1, b-a}:=X_{1, b-a}^{+}\left(n_{p}\right) \cdots X_{1, b-a}^{+}\left(n_{1}\right) v_{a-1, b-1}, \quad \text { if } b-1=M+N\right) . \\
\text { If } X_{i}^{+}(m) v_{a-1, b-1}=X_{k, l}^{+}(m) v_{a-1, b-1}=0, \forall i \neq M, m \in \mathbb{Z},(k, l)>(a-1, b-1), \text { then } \\
X_{i}^{+}(m) v_{a, b}=X_{k, l}^{+}(m) v_{a, b}=0 \quad \forall m \in \mathbb{Z},(k, l)>(a, b), i \neq M, \\
\text { (resp. } \left.X_{i}^{+}(m) v_{1, b-a}=X_{k, l}^{+}(m) v_{1, b-a}=0 \quad \forall m \in \mathbb{Z},(k, l)>(1, b-a)\right) .
\end{gathered}
$$

Proof. See Appendix B.

Lemma 3.7. For $(a, b) \in S$, let $v_{a, b}$ be a weight vector in $V$ such that

$$
\begin{aligned}
& X_{i}^{+}(m)\left(X_{a, b}^{+}\left(n_{1}\right) \cdots X_{a, b}^{+}\left(n_{r}\right) v_{a, b}\right)=0, \quad \forall m, n_{1}, \ldots, n_{r} \in \mathbb{Z}, i \neq M, \\
& X_{a, b}^{+}(p) X_{a, b}^{+}(k) v_{a, b}=0, \quad \forall p, k \in \mathbb{Z} \text { with } p \equiv k(\bmod 2) .
\end{aligned}
$$

Then

$$
X_{a, b}^{+}(p) X_{a, b}^{+}(i) X_{a, b}^{+}(j) v_{a, b}=0, \quad \forall p, i, j \in \mathbb{Z}
$$


Furthermore, there exists $k(0 \leq k \leq 2)$ and $n_{1}, \ldots, n_{k} \in \mathbb{Z}$ such that

$$
\begin{aligned}
& w_{a, b}:=X_{a, b}^{+}\left(n_{1}\right) \cdots X_{a, b}^{+}\left(n_{k}\right) v_{a, b} \neq 0, \\
& X_{a, b}^{+}(m) w_{a, b}=0, \quad \forall m \in \mathbb{Z} .
\end{aligned}
$$

Proof. See Appendix C.

Let $V$ be an irreducible zero-level integrable module for $\mathrm{U}_{q}$ with finite dimensional weight spaces. By definition, $V$ is integrable over the even subalgebra of $\mathrm{U}_{q}$. It follows from Chari's work [6] that there is a non-zero weight vector $v \in V$ such that

$$
X_{i}^{+}(m) v=0, \quad \forall m \in \mathbb{Z}, i \neq M .
$$

Denote by $w t(v)$ the weight of $v$. Let $X$ be the subspace of $V$ spanned by the vectors $X_{M}^{+}(k) X_{M}^{+}(-k) v$ for all $k \geq 0$, which is a subspace of $V_{w t}(v)+2 \alpha_{M}$, thus is finite dimensional. Therefore, there exists a finite positive integer $K$ such that

$$
X=\operatorname{span}\left\{X_{M}^{+}(k) X_{M}^{+}(-k) v \mid 0<k<K\right\} .
$$

Thus for any $r \in \mathbb{Z}$ we have

$$
X_{M}^{+}(r) X_{M}^{+}(-r) v=\sum_{0<k<K} a_{k}^{(r)} X_{M}^{+}(k) X_{M}^{+}(-k) v, \quad a_{k}^{(r)} \in \mathbb{C} .
$$

Note that the elements $X_{M}^{+}(k)$ for all $k \in \mathbb{Z}$ anti-commute among themselves and satisfy $X_{M}^{+}(k)^{2}=0$. Thus equation (3.16) below immediately follows from (3.15).

Lemma 3.8. Let $V$ be a simple zero-level integrable $\mathrm{U}_{q}$-module, and let $v \in V$ be a nonzero weight vector satisfying (3.14). Then the following relations hold for large $k$ :

$$
\begin{aligned}
& X_{M}^{+}\left(n_{k}\right) X_{M}^{+}\left(-n_{k}\right) \cdots X_{M}^{+}\left(n_{1}\right) X_{M}^{+}\left(-n_{1}\right) v=0, \quad \forall n_{1}, \ldots, n_{k} \in \mathbb{Z} ; \\
& X_{1, M+N-1}^{+}\left(m_{k}\right) \cdots X_{1, M+N-1}^{+}\left(m_{1}\right) v=0, \quad \forall m_{1}, \ldots, m_{k} \in \mathbb{Z} .
\end{aligned}
$$

Proof. Since (3.16) was proven already, we only need to consider (3.17). For notational simplicity, we write $E(m)=X_{1, M+N-1}^{+}(m)$ for all $m$. Applying

$$
\left(X_{M+N-1}^{+}(0)\right)^{2 k} \cdots\left(X_{M+1}^{+}(0)\right)^{2 k}\left(X_{1}^{+}(m)\right)^{2 k}\left(X_{2}^{+}(0)\right)^{2 k} \cdots\left(X_{M-1}^{+}(0)\right)^{2 k}
$$

to (3.16) and then using (A.2), we can show that

$$
E\left(m+n_{k}\right) E\left(m-n_{k}\right) \cdots E\left(m+n_{1}\right) E\left(m-n_{1}\right) v=0 .
$$

Let $l+1$ be the minimal integer such that (3.18) holds. Then there exist $r_{1}, \cdots, r_{l}$ such that

$$
\begin{aligned}
& v^{\prime}:=E\left(m+r_{l}\right) E\left(m-r_{l}\right) \cdots E\left(m+r_{1}\right) E\left(m-r_{1}\right) v \neq 0 \\
& E(p) E(k) v^{\prime}=0 \quad \text { for all } p, k \in \mathbb{Z} \text { with } p \equiv k(\bmod 2) .
\end{aligned}
$$

By Lemma 3.6, we have

$$
X_{i}^{+}(m)\left(E\left(n_{1}\right) \cdots E\left(n_{k}\right) v^{\prime}\right)=0, \quad i \neq M, n_{1}, \ldots, n_{k} \in \mathbb{Z}, k \in \mathbb{Z}_{\geq 0} .
$$

Now (3.17) follows from 3.13). 
Using Lemma 3.8, we can prove the following result.

Proposition 3.9. Let $V$ be an irreducible zero-level integrable $\mathrm{U}_{q}$-module with finite dimensional weight spaces. Then there always exists a nonzero weight vector $w \in V$ such that

$$
\begin{aligned}
& X_{i}^{+}(r) w=0, \quad \forall i \neq M, r \in \mathbb{Z}, \\
& X_{a, b}^{+}(r) w=0, \quad \forall(a, b) \in S, r \in \mathbb{Z}, \\
& X_{M}^{+}\left(n_{1}\right) \cdots X_{M}^{+}\left(n_{k}\right) w=0, \quad \forall n_{i} \in \mathbb{Z}, \text { large } k .
\end{aligned}
$$

Proof. By Lemma 3.8, one can find a non-zero weight vector $v_{1, M+N-1}$ such that

$$
X_{i}^{+}(m) v_{1, M+N-1}=X_{1, M+N-1}^{+}(m) v_{1, M+N-1}=0 \quad i \neq M, m \in \mathbb{Z} .
$$

We observe that (3.16) still holds if we replace $v$ by $v_{1, M+N-1}$, namely, for large $k$,

$$
X_{M}^{+}\left(n_{k}\right) X_{M}^{+}\left(-n_{k}\right) \cdots X_{M}^{+}\left(n_{1}\right) X_{M}^{+}\left(-n_{1}\right) v_{1, M+N-1}=0, \quad \forall n_{1}, \ldots, n_{k} \in \mathbb{Z} .
$$

Applying $\left(X_{M+N-2}^{+}(0)\right)^{2 k} \cdots\left(X_{M+1}^{+}(0)\right)^{2 k}\left(X_{1}^{+}(m)\right)^{2 k}\left(X_{2}^{+}(0)\right)^{2 k} \cdots\left(X_{M-1}^{+}(0)\right)^{2 k}$ to this equation, and then using (A.2), we obtain

$$
X_{1, M+N-2}^{+}\left(m+n_{k}\right) X_{1, M+N-2}^{+}\left(m-n_{k}\right) \cdots X_{1, M+N-2}^{+}\left(m+n_{1}\right) X_{1, M+N-2}^{+}\left(m-n_{1}\right) v=0 \text {. }
$$

Clearly, $m+n_{i} \equiv m-n_{i}(\bmod 2), i=1, \ldots, k$. From Lemma 3.6 and Lemma 3.7, we can find a non-zero weight vector $v_{1, M+N-2}$ such that for all $m \in \mathbb{Z}$,

$$
\begin{aligned}
& X_{i}^{+}(m) v_{1, M+N-2}=0, \quad i \neq M, \\
& X_{1, M+N-1}^{+}(m) v_{1, M+N-2}=X_{1, M+N-2}^{+}(m) v_{1, M+N-2}=0 .
\end{aligned}
$$

Repeating the above arguments for a finite number of times, we will find a nonzero weight vector $w$ such that

$$
X_{i}^{+}(m) w=X_{a, b}^{+}(m) w=0, \quad i \neq M, m \in \mathbb{Z},(a, b) \in S .
$$

Let $\mu$ be the weight of $w$. Observe that $V$, being irreducible, must be cyclically generated by $w$ over $\mathrm{U}_{q}$. By using the PBW theorem for $\mathrm{U}_{q}$ and equation (3.23), we easily show that any weight of $V$ which is bigger than $\mu$ (relative to $B$; see also the Borel subalgebra of $\widehat{\mathfrak{s l}}(M \mid N)$ defined by (2.2) ) must be of the form

$$
\mu+a\left(\varepsilon_{M}-\varepsilon_{M+1}\right)+b \delta, \quad a \in \mathbb{Z}_{\geq 0}, b \in \mathbb{Z} .
$$

Now we prove (3.21). Suppose it is false, that is, for any positive integer $p$, there always exist $k>p$ and $n_{1}, \ldots, n_{k} \in \mathbb{Z}$ such that $\tilde{w}:=X_{M}^{+}\left(n_{1}\right) \cdots X_{M}^{+}\left(n_{k}\right) w \neq 0$. Then $v:=\mu+k\left(\varepsilon_{M}-\varepsilon_{M+1}\right)+\sum_{i=1}^{k} n_{i} \delta$ is the weight of $\tilde{w}$. But for large $p$, and hence large $k$, we have $\left(v, \varepsilon_{M-1}-\varepsilon_{M}\right)<0$. Thus $v+\left(\varepsilon_{M-1}-\varepsilon_{M}\right)$ is a weight of $V$ by considering the action of the $\mathrm{U}_{q}\left(\mathfrak{s l}_{2}\right)$ subalgebra generated by $X_{M-1}^{ \pm}(0)$ and $K_{M-1}^{ \pm 1}$. However, the weight $v+\left(\varepsilon_{M-1}-\varepsilon_{M}\right)$ is not of the form (3.24), proving (3.21) by contradiction.

The following theorem is now an easy consequence of Proposition 3.9 
Theorem 3.10. Let $V$ be an irreducible zero-level integrable $\mathrm{U}_{q}$-module with finite dimensional weight spaces. Then $V$ is a highest weight module with respect to the triangular decomposition of $\mathrm{U}_{q}$ given by (2.3).

Proof. Consider the weight vector $w$ of Proposition 3.9. and let $s$ be the minimal integer such that (3.21) holds. Then there exist $r_{1}, \ldots, r_{s-1} \in \mathbb{Z}$ such that

$$
\begin{aligned}
& v:=X_{M}^{+}\left(r_{1}\right) \cdots X_{M}^{+}\left(r_{s-1}\right) w \neq 0, \\
& X_{M}^{+}(r) v=0, \quad \forall r \in \mathbb{Z} .
\end{aligned}
$$

It it not difficult to show that we also have $X_{i}^{+}(m) v=0$ for all $i \neq M$ and $m \in \mathbb{Z}$.

Theorem 3.11. Let $W$ be an irreducible integrable $\mathrm{U}_{q}$-module of type 1 with finite dimensional weight spaces. Then $W$ is isomorphic to an irreducible component of $L(V, b)$ for some $b \in \mathbb{C}$, where $V=V(P, f, c, Q)$ (see Definition 3.3) for some $(P, f, c, Q) \in$ $\mathcal{R}_{M, N}$.

Proof. It follows from Theorem 3.10 that there exists a nonzero highest weight vector $v \in W$, and $W=\mathrm{U}_{q} v=\mathrm{U}_{q}^{-} \mathrm{U}_{q}(0) v$, where the second equality follows from (2.3). Clearly $\mathrm{U}_{q}(0) v=\mathrm{U}_{q}^{\prime}(0) v$. Irreducibility of $W$ requires that $\mathrm{U}_{q}^{\prime}(0) v$ be an irreducible $\mathrm{U}_{q}(0)$-module, and hence an irreducible $\mathrm{U}_{q}^{\prime}(0)$-module. Since $\mathrm{U}_{q}^{\prime}(0)$ is a $\mathbb{Z}$-graded commutative algebra, $\mathrm{U}_{q}^{\prime}(0) v$ being an irreducible graded module must be the quotient of $\mathrm{U}_{q}^{\prime}(0)$ by a maximal graded ideal $\mathcal{M}$ of $\mathrm{U}_{q}^{\prime}(0)$ which annihilates $v$. It follows from Lemma 3.4 that $\mathrm{U}_{q}^{\prime}(0) / \mathcal{M} \cong L_{r}:=\mathbb{C}\left[t^{r}, t^{-r}\right]$. Thus we have a natural $\mathbb{Z}$-graded homomorphism $\tilde{\varphi}: \mathrm{U}_{q}^{\prime}(0) \rightarrow \mathrm{U}_{q}^{\prime}(0) / \mathcal{M} \cong L_{r}$ such that $\tilde{\varphi}(x) v=x v$ for all $x \in \mathrm{U}_{q}^{\prime}(0)$. There exists some $b \in \mathbb{C}$ such that $D v=q^{b} v$. Set $\varphi=(\tilde{\varphi}, b)$ (see notation immediately below (3.7)). Then $W$ is isomorphic to $V(\varphi)$.

Set $\psi=S \circ \tilde{\varphi}$ and consider the irreducible module $V(\psi)$. By Theorem 3.5, $V(\varphi)$ is isomorphic to an irreducible component of $L(V(\psi), b)$. Since $V(\varphi)$ is an integrable $\mathrm{U}_{q}$-module with finite dimensional weight spaces, so is $V(\psi)$. Thus it follows from Theorem 3.2 that $V(\psi)$ is isomorphic to $V(P, f, c, Q)$ (see Definition 3.3) for some $(P, f, c, Q) \in \mathcal{R}_{M, N}$. This completes the proof.

\section{INTEGRABLE REPRESENTATIONS AT NONZERO LEVELS}

In this section, highest and lowest weight $\widehat{U}_{q}$-modules are defined relative to the triangular decomposition of $\widehat{\mathrm{U}}_{q}$ given in Proposition 2.2.

4.1. The subalgebra of $\widehat{\mathrm{U}}_{q}$ generated by $X_{i}^{ \pm}(n), K_{i}^{ \pm 1}, h_{i}(r), C^{ \pm 1 / 2}, D^{ \pm 1}$ (with $M+1 \leq$ $i \leq M+N-1, n, r \in \mathbb{Z}, r \neq 0)$ is $\mathrm{U}_{q^{-1}}(\widehat{\mathfrak{s l}}(N))$. Thus $C$ acts on any nontrivial simple integrable highest weight $\mathrm{U}_{q^{-1}}(\widehat{\mathfrak{s l}}(N))$-module $[8,18]$ by the multiplication by $q^{-\ell}$ for some fixed $\ell>0$.

We have the following result. 
Proposition 4.1. (1) Let $W$ be an integrable $\mathrm{U}_{q^{-1}}(\widehat{\mathfrak{s l}}(N))$-module with finite dimensional weight spaces. Suppose that the center $C$ acts on $W$ by $\left(q^{-1}\right)^{r}$ with $r \in \mathbb{Z}_{>0}$. If $\lambda$ is a weight of $W$, then there exists $K>0$ such that

$$
\lambda+\alpha+k \delta \text { is not a weight of } W \text { for all } k \geq K \text { and } \alpha \in \Delta_{0}^{2} \cup\{0\} .
$$

(2) Let $V$ be an irreducible integrable module for $\widehat{\mathrm{U}}_{q}$ with finite dimensional weight spaces. Suppose that the center $C$ acts on $V$ by $\left(q^{-1}\right)^{r}$ with $r \in \mathbb{Z}_{>0}$. Then for any $\lambda \in P(V)$ there exists $K>0$ such that

$$
\lambda+\alpha+k \delta \notin P(V) \quad \text { for all } k \geq K \text { and for all } \alpha \in \Delta_{0}^{2} \cup\{0\} .
$$

Proof. Part (1) can be easily proved by adapting the proof of [24, Theorem 1.10] to the present context. We omit the details.

To prove part (2), set $T=\mathbb{C}\left[\left(K_{M}^{N} K_{M+1}^{N-1} \cdots K_{M+N-2}^{2} K_{M+N-1}\right)^{ \pm 1}, K_{M-1}^{ \pm 1}, \ldots, K_{1}^{ \pm 1}\right]$. Observe that $T$ commutes with $\mathrm{U}_{q^{-1}}(\widehat{\mathfrak{s l}}(N))$. Decompose $V$ into the direct sum of $T$ invariant subspaces. Each $T$-invariant subspace is an integrable $\mathrm{U}_{q^{-1}}(\widehat{\mathfrak{s l}}(N))$-module with finite dimensional weight spaces. Now part (1) implies part (2).

Theorem 4.2. Assume that both $M$ and $N$ are greater than 1 . Then there exists no integrable $\widehat{\mathrm{U}}_{q}$-module with finite dimensional weight spaces, where $C$ does not act by the identity.

Proof. Without lose of generality, we may assume that $C$ acts by $q^{-r}$ with $r>0$. Let $\mathrm{U}_{q}(\widehat{\mathfrak{s l}}(M))$ be the subalgebra of $\widehat{\mathrm{U}}_{q}$ generated by $X_{i}^{ \pm}(n), K_{i}^{ \pm 1}, h_{i}(s), C^{ \pm 1 / 2}$ with $1 \leq i \leq$ $M-1, n, s \in \mathbb{Z}$ and $s \neq 0$. Regard $V$ as an integrable $\widehat{\mathrm{U}}_{q}(\mathfrak{s l}(M))$-module. Note that $C$ acts on $V$ by $q^{-r}$ with $r>0$. By [6, Theorem 5], there exists a weight vector $v \in V$ of weight $\lambda$ such that $X_{i}^{ \pm}(n) v=0$ and $h_{i}(n) v=0$ for all $n<0$ and $1 \leq i \leq M-1$. Then $h_{i}(n) v \neq 0$ for all $n>0$ and $1 \leq i \leq M-1$. Thus $\lambda+n \delta \in P(V)$ for all $n>0$. This contradicts Proposition 4.1, completing the proof.

Remark 4.3. A similar result has long been [12, 15] for affine Lie superalgebras in the classical setting.

4.2. From now on we assume that $N>M=1$.

Lemma 4.4. $X_{1, a}^{ \pm}(m) X_{1, a}^{ \pm}(n)=-X_{1, a}^{ \pm}(n) X_{1, a}^{ \pm}(m), \quad 1 \leq a \leq N-1, m, n \in \mathbb{Z}$.

Proof. The $a=1$ case is a defining relation of $\widehat{\mathrm{U}}_{q}$. For $a \geq 2$, we have

$$
\left[X_{1, a}^{+}(m), X_{1, a}^{+}(n)\right]_{q^{-2}}=\left[\left[X_{1}^{+}(m), X_{2, a}^{+}(0)\right]_{q^{-1}},\left[X_{1}^{+}(n), X_{2, a}^{+}(0)\right]_{q^{-1}}\right]_{q^{-2}} .
$$

We can rewrite the right hand side as

$$
\left[X_{1}^{+}(m),\left[X_{2, a}^{+}(0), X_{1, a}^{+}(n)\right]_{q^{-1}}\right]_{q^{-2}}+q^{-1}\left[\left[X_{1}^{+}(m),\left[X_{1}^{+}(n), X_{2, a}^{+}(0)\right]_{q^{-1}}\right]_{q^{-1}}, X_{2, a}^{+}(0)\right],
$$


where the first term vanishes by Lemma A.2. The second term can be expressed as

$$
\begin{aligned}
& q^{-1}\left[\left[\left[X_{1}^{+}(m), X_{1}^{+}(n)\right], X_{2, a}^{+}\right]_{q^{-2}}, X_{2 . a}^{+}(0)\right] \\
& -q^{-1}\left[\left[X_{1}^{+}(n),\left[X_{1}^{+}(m), X_{2, a}^{+}(0)\right]_{q^{-1}}\right]_{q^{-1}}, X_{2 . a}^{+}(0)\right],
\end{aligned}
$$

where the first term vanishes, as $\left[X_{1}^{+}(m), X_{1}^{+}(n)\right]=0$. By manipulating the second term, we obtain

$$
\begin{aligned}
{\left[X_{1, a}^{+}(m), X_{1, a}^{+}(n)\right]_{q^{-2}}=} & -q^{-1}\left(\left[X_{1}^{+}(n),\left[X_{1, a}^{+}(m), X_{2, a}^{+}(0)\right]_{q}\right]_{q^{-2}}\right. \\
& +q\left[\left[X_{1}^{+}(n), X_{2, a}^{+}(0)\right]_{q^{-1}},\left[X_{1}^{+}(m), X_{2, a}^{+}(0)\right]_{q^{-1}}\right]_{q^{-2}} \\
= & -\left[X_{1, a}^{+}(n), X_{1, a}^{+}(m)\right]_{q^{-2}} .
\end{aligned}
$$

Hence, $X_{1, a}^{+}(m) X_{1, a}^{+}(n)=-X_{1, a}^{+}(n) X_{1, a}^{+}(m)$.

Similarly, one can show that $X_{1, a}^{-}(m) X_{1, a}^{-}(n)=-X_{1, a}^{-}(n) X_{1, a}^{-}(m)$.

Theorem 4.5. Assume that $N>M=1$. Let $V$ be an irreducible integrable $\widehat{\mathrm{U}}_{q}$-module with finite dimensional weight spaces. Suppose that $C$ acts by $\left(q^{-1}\right)^{r}$ for some non-zero $r \in \mathbb{Z}$. If $r>0$ (resp. $r<0$ ), then $V$ is a highest (resp. lowest) weight module.

Proof. Without lose of generality, we may assume that $r>0$.

Claim 1. For any weight vector $v \in V$, the following vector space, spanned by

$$
\left\{\begin{array}{l|l}
X_{1, a_{1}}^{+}\left(m_{1}\right) \cdots X_{1, a_{k}}^{+}\left(m_{k}\right) v \mid \begin{array}{l}
1 \leq a_{1} \leq \cdots \leq a_{k} \leq N, k \geq 0 \\
m_{i} \geq 0, m_{i}<m_{i+1} \text { when } a_{i}=a_{i+1}
\end{array}
\end{array}\right\}
$$

is finite-dimensional.

By Proposition 2.3, it is sufficient to prove that, for $1 \leq p \leq N$, the vector space $S_{p}^{+}(v)$ spanned by $\left\{X_{1, p}^{+}\left(m_{1}\right) \cdots X_{1, p}^{+}\left(m_{r}\right) v \mid r \in \mathbb{Z}_{\geq 0}, m_{i} \geq 0\right\}$ is finite-dimensional.

For $S_{1}^{+}(v)$, which is spanned by $\left\{X_{1}^{+}\left(m_{1}\right) \cdots X_{1}^{+}\left(m_{r}\right) v \mid 0 \leq m_{1}<\cdots<m_{r}, r \in \mathbb{Z}_{\geq 0}\right\}$, we consider

$$
\begin{aligned}
X_{1}^{+}(n) v & =\frac{n\left(q-q^{-1}\right)}{q^{-n}-q^{n}} C^{\frac{|n|}{2}}\left[h_{2}(n), X_{1}^{+}(0)\right] v \\
& =\frac{n\left(q-q^{-1}\right)}{q^{-n}-q^{n}} C^{\frac{|n|}{2}}\left(h_{2}(n) X_{1}^{+}(0) v-X_{1}^{+}(0) h_{2}(n) v\right) .
\end{aligned}
$$

From Proposition 4.1 there exists $n_{0}>0$ such that $h_{2}(n) v=0$ and $h_{2}(n) X_{1}^{+}(0) v=0$ for all $n>n_{0}$. Now it is easy to see that $S_{1}^{+}(v)$ is spanned by

$$
\left\{X_{M}^{+}\left(m_{1}\right) \cdots X_{M}^{+}\left(m_{r}\right) v \mid r \geq 0,0 \leq m_{i} \leq n_{0}, m_{i} \neq m_{j}, i \neq j\right\},
$$

which is clearly finite-dimensional.

For any $r \geq 1$ and $m_{i} \geq 0$, observe that

$$
X_{1}^{+}\left(m_{1}\right) \cdots X_{1}^{+}\left(m_{r}\right) v=0
$$


if there exists $j(1 \leq j \leq r)$ such that $m_{j}>n_{0}$. From Proposition 4.1 there exists $K_{v}>0$ such that $X_{i}^{+}(k) v=0$ for all $k \geq K_{v}, 2 \leq i \leq N$. Applying $\left(X_{p}^{+}\left(K_{v}\right)\right)^{r} \cdots\left(X_{M-1}^{+}\left(K_{v}\right)\right)^{r}$ to (4.1), and using (A.2) (A.4) repeatedly, we have

$$
X_{1, p}^{+}\left(m_{1}+(M-p) K_{v}\right) \cdots X_{1, p}^{+}\left(m_{r}+(M-p) K_{v}\right) v=0 .
$$

if there exists $j(1 \leq j \leq r)$ such that $m_{j}>n_{0}$. Combining this with Lemma 4.4, we conclude that the vector space $S_{p}^{+}(v)$ is spanned by

$$
\left\{X_{1, p}^{+}\left(m_{1}\right) \cdots X_{1, p}^{+}\left(m_{r}\right) v \mid 0 \leq m_{1}<\cdots<m_{r} \leq(M-p) K_{v}+n_{0}, r \in \mathbb{Z}_{\geq 0}\right\},
$$

which is finite-dimensional. This completes the proof of Claim 1.

In a similar way, one can prove

Claim 2. For any weight vector $v \in V$, the vector space spanned by the following set

is finite-dimensional.

$$
\left\{\begin{array}{l|l}
X_{1, a_{1}}^{-}\left(m_{1}\right) \cdots X_{1, a_{k}}^{-}\left(m_{k}\right) v \mid \begin{array}{l}
1 \leq a_{1} \leq \cdots \leq a_{k} \leq N, k \geq 0 \\
m_{i}>0, m_{i}<m_{i+1} \text { when } a_{i}=a_{i+1}
\end{array}
\end{array}\right\},
$$

Let $\mathcal{N}^{+}$(resp. $\mathcal{N}^{-}$) be the subalgebra of $\widehat{\mathrm{U}}_{q}$ generated by $X_{1, a}^{+}(0), X_{1, a}^{ \pm}(n), n>0,1 \leq$ $a \leq N$ (resp. $\left.\quad X_{1, a}^{+}(0), X_{1, a}^{ \pm}(n), n<0,1 \leq a \leq N\right)$. Combining Proposition 2.3 with Claims 1 and 2, we obtain

Claim 3. For any weight vector $v \in V$, the space $\mathcal{N}^{+} v$ is finite-dimensional.

For any weight vector $v \in V$, set $W=\mathbb{C}\left[K_{1}, K_{1}^{-1}\right] \mathrm{U}\left(\bigoplus_{n>0} \mathbb{C} h_{1}(n)\right) \mathcal{N}^{+} v$. From Proposition 4.1 and Claim 3, one can see that $W$ is finite-dimensional.

Define the following subalgebras of $\widehat{\mathrm{U}}_{q}$ :

- $\mathrm{U}_{q^{-1}}(\widehat{\mathfrak{s l}}(N))$ is generated by $X_{i}^{ \pm}(n), h_{i}(r), K_{i}^{ \pm 1}, C^{ \pm 1 / 2}, D^{ \pm 1}, n, r \in \mathbb{Z}, r \neq 0,2 \leq$ $i \leq N$

- $\widehat{\mathrm{U}}_{q^{-1}, N}^{+}$by $\left\{X_{i}^{+}(0), X_{i}^{ \pm}(n), h_{i}(n) \mid n>0,2 \leq i \leq N\right\}$;

- $\widehat{\mathrm{U}}_{q^{-1}, N}^{-}$by $\left\{X_{i}^{-}(0), X_{i}^{ \pm}(n), h_{i}(n) \mid n<0,2 \leq i \leq N\right\}$; and

- $\widehat{\mathrm{U}}_{q^{-1, N}}^{0}$ by $\left\{K_{i}^{ \pm 1}, C^{ \pm 1 / 2}, D^{ \pm 1} \mid 2 \leq i \leq N\right\}$.

Now consider $\mathcal{W}=\widehat{\mathrm{U}}_{q^{-1}, N}^{-} \widehat{\mathrm{U}}_{q^{-1}, N}^{0} \widehat{\mathrm{U}}_{q^{-1}, N}^{+} W$. Clearly, $\mathcal{W}$ is an integrable $\mathrm{U}_{q^{-1}}(\widehat{\mathfrak{s l}}(N))$ module. Using proposition 4.1, one can show that $\widehat{\mathrm{U}}_{q^{-1}, N}^{+} W$ is finite-dimensional. By [6. Proposition 1.7], we have $\mathcal{W} \cong \bigoplus_{\lambda} m_{\lambda} V(\lambda)$, where $V(\lambda)$ are irreducible integrable $\mathrm{U}_{q^{-1}}(\widehat{\mathfrak{s l}}(N))$-modules with highest weight $\lambda$ and multiplicities $m_{\lambda} \in \mathbb{Z}_{\geq 0}$, which are nonzero for only finitely many $\lambda$. Thus $\mathcal{W}$ has a maximal weight. Since $V$ is simple, $V=\mathcal{N}^{-} \mathrm{U}\left(\underset{n<0}{\bigoplus} \mathbb{C} h_{1}(n)\right) \mathcal{W}$. Thus this maximal weight of $\mathcal{W}$ is also the highest weight of $V$. 
Recall that for ordinary quantum affine algebras, a simple highest (resp. lowest) weight module is integrable if and only if its highest (resp. lowest) weight is integral dominant (resp. anti-dominant) [8, 18].

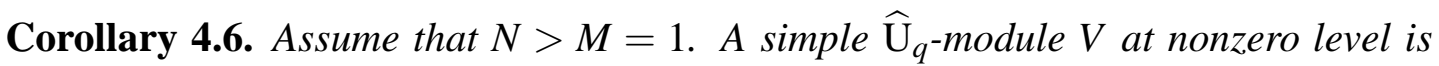
integrable with finite dimensional weight spaces if and only if $V$ is

(1) a highest weight module with a highest weight which is integral dominant with respect to $\mathrm{U}_{q}\left(\widehat{\mathfrak{s l}}(M \mid N)_{\overline{0}}\right)$, or

(2) a lowest weight module with a lowest weight which is integral anti-dominant with respect to $\mathrm{U}_{q}\left(\widehat{\mathfrak{s l}}(M \mid N)_{\overline{0}}\right)$.

Proof. As simple highest or lowest weight $\widehat{\mathrm{U}}_{q}$-modules defined with respect to the triangular decomposition of $\widehat{U}_{q}$ given in Proposition 2.2 automatically have finite dimensional weight spaces, the corollary immediately follows from Theorem 4.5 and the preceding remarks on integrable highest weight modules for ordinary quantum affine algebras.

\section{APPENDiX A. RELATIONS IN $\mathrm{U}_{q}$}

We present some technical results which are used in the main body of the paper.

The following identities are valid for the $q$-bracket.

Lemma A.1. For any homogeneous elements $a, b, c$ of $\widehat{\mathrm{U}}_{q}$, and nonzero scalars $u, v, x$,

$$
\begin{aligned}
& {[a, b c]_{v}=[a, b]_{x} c+(-1)^{|a||b|_{x}} x[a, c]_{\frac{v}{x}},} \\
& {[a b, c]_{v}=a[b, c]_{x}+(-1)^{|b||c|} x[a, c]_{\frac{v}{x}} b,} \\
& {\left[a,[b, c]_{u}\right]_{v}=\left[[a, b]_{x}, c\right]_{\frac{u v}{x}}+(-1)^{|a||b|} x\left[b,[a, c]_{\frac{v}{x}}\right]_{\frac{u}{x}},} \\
& {\left[[a, b]_{u}, c\right]_{v}=\left[a,[b, c]_{x}\right]_{\frac{u v}{x}}+(-1)^{|b||c|} x\left[[a, c]_{\frac{v}{x}}, b\right]_{\frac{u}{x}} .}
\end{aligned}
$$

We can derive from Definition 2.1 the following relations:

$$
\begin{gathered}
{\left[\left[X_{i}^{+}(m),\left[X_{i}^{+}(m), X_{j}^{+}(k)\right]_{q^{-1}}\right]_{q}\right.} \\
=\left[\left[X_{i}^{+}(m),\left[X_{i}^{+}(m), X_{j}^{+}(k)\right]_{q}\right]_{q^{-1}}=0, \quad i \neq M, a_{i, j}= \pm 1,\right. \\
{\left[\left[X_{M}^{+}(m),\left[X_{M}^{+}(m), X_{j}^{+}(k)\right]_{q^{-1}}\right]_{q^{-1}}\right.} \\
=\left[X_{M}^{+}(m),\left[X_{M}^{+}(m), X_{j}^{+}(k)\right]_{q}\right]_{q}=0, \quad a_{M, j}= \pm 1 . \\
{\left[X_{i-1}^{+}(m), X_{i}^{+}(n)\right]_{q_{i}}=q_{i}^{k}\left[X_{i-1}^{+}(m+k), X_{i}^{+}(n-k)\right]_{q_{i}}} \\
\quad+\sum_{s=1}^{k} q_{i}^{s-1}\left(q_{i}^{2}-1\right) X_{i}^{+}(n-s) X_{i-1}^{+}(m+s),
\end{gathered}
$$




$$
\begin{aligned}
{\left[X_{i-1}^{+}(m), X_{i}^{+}(n)\right]_{q_{i}} } & =q_{i}^{-k}\left[X_{i-1}^{+}(m-k), X_{i}^{+}(n+k)\right]_{q_{i}} \\
& -\sum_{s=0}^{k-1} q_{i}^{-s-1}\left(q_{i}^{2}-1\right) X_{i}^{+}(n+s) X_{i-1}^{+}(m-s) .
\end{aligned}
$$

$$
\begin{aligned}
{\left[X_{M}^{+}(m), X_{M+1}^{+}(n)\right]_{q} } & =q^{k}\left[X_{M}^{+}(m+k), X_{M+1}^{+}(n-k)\right]_{q} \\
& +\sum_{s=0}^{k-1} q^{s}\left(1-q^{2}\right) X_{M}^{+}(m+s) X_{M+1}^{+}(n-s) .
\end{aligned}
$$

$$
\begin{aligned}
{\left[X_{i+1}^{+}(m), X_{i}^{+}(n)\right]_{q} } & =q^{k}\left[X_{i+1}^{+}(m-k), X_{i}^{+}(n+k)\right]_{q} \\
& +\sum_{s=1}^{k} q^{s-1}\left(q^{2}-1\right) X_{i}^{+}(n+s) X_{i+1}^{+}(m-s), \quad i \geq M
\end{aligned}
$$

(A.8) $\left[X_{i+1}^{+}(m), X_{i}^{+}(n)\right]_{q}=q^{-k}\left[X_{i+1}^{+}(m+k), X_{i}^{+}(n-k)\right]_{q}$

$$
-\sum_{s=0}^{k-1} q^{-s-1}\left(q^{2}-1\right) X_{i}^{+}(n-s) X_{i+1}^{+}(m+s), \quad i \geq M .
$$

Combining (A.4) with (A.5), (A.7) with A.8), respectively, we have

$$
\begin{aligned}
{\left[X_{i-1}^{+}(m), X_{i}^{+}(n)\right]_{q_{i}} } & =q_{i}^{ \pm k}\left[X_{i-1}^{+}(m \pm k), X_{i}^{+}(n \mp k)\right]_{q_{i}} \\
& +\sum_{s=0}^{|k|} c_{s} X_{i}^{+}(n \mp s) X_{i-1}^{+}(m \pm s) \quad c_{s} \in \mathbb{C}
\end{aligned}
$$

$$
\begin{aligned}
{\left[X_{i+1}^{+}(m), X_{i}^{+}(n)\right]_{q} } & =q^{ \pm k}\left[X_{i+1}^{+}(m \mp k), X_{i}^{+}(n \pm k)\right]_{q} \\
& +\sum_{s=0}^{|k|} c_{s} X_{i}^{+}(n \pm s) X_{i+1}^{+}(m \mp s), \quad i \geq M, c_{s} \in \mathbb{C}
\end{aligned}
$$

We have the following result.

Lemma A.2. (1) $\left[\left[\left[X_{i-1}^{+}(m), X_{i}^{+}(n)\right]_{q_{i}}, X_{i+1}^{+}(k)\right]_{q_{i+1}}, X_{i}^{+}(n)\right]=0, \quad i \neq M$.

(2) $\left[X_{i}^{+}(0), X_{a, b}^{+}(n)\right]=0, \quad a<i<b, n \in \mathbb{Z}$.

(3) $\left[X_{M}^{+}(0), X_{a, M}^{+}(n)\right]_{q^{-1}}=0, \quad a<M, n \in \mathbb{Z}$.

(4) $\left[X_{b}^{+}(0), X_{a, b}^{+}(n)\right]_{q_{b}}=0, \quad b \neq M, n \in \mathbb{Z}$.

Proof. Part (1) can be found in [30, Lemma 6.1.1]. Part (2) follows from (1), part (3) follows from (A.3), and part (4) follows from (A.2). 
Appendix B. Proof of Lemma 3.6

Proof of Lemma 3.6 Set $v_{a, b}^{(t-1)}:=X_{a, b}^{+}\left(n_{t-1}\right) \cdots X_{a, b}^{+}\left(n_{1}\right) v_{a-1, b-1}, t=1, \ldots, p+1$. We use induction on $t$ starting from the given case $t=1$. Assume that

$$
X_{i}^{+}(m) v_{a, b}^{(t-1)}=X_{k, l}^{+}(m) v_{a, b}^{(t-1)}=0 \text { for all } i \neq M, m \in \mathbb{Z},(k, l)>(a, b) .
$$

First we want to prove that $X_{i}^{+}(m) v_{a, b}^{(t)}=0$ for all $i \neq M$.

If $i>b+1$ or $i<a-1$, then $\left[X_{i}^{+}(m), X_{a, b}^{+}(n)\right]=0$. Thus $X_{i}^{+}(m) v_{a, b}^{(t)}=0$.

If $i=a-1$, we have

$$
\begin{aligned}
X_{a-1}^{+}(m) v_{a, b}^{(t)} & =\left[X_{a-1}^{+}(m), X_{a, b}^{+}\left(n_{t}\right)\right]_{q_{a}} v_{a, b}^{(t-1)} \\
& =\left[\left[X_{a-1}^{+}(m), X_{a}^{+}\left(n_{t}\right)\right]_{q_{a}}, X_{a+1, b}^{+}(0)\right]_{q_{a+1}} v_{a, b}^{(t-1)}
\end{aligned}
$$

Using equation (A.9), we can rewrite the right hand side as

$$
\begin{aligned}
& \left.q_{a}^{n_{t}}\left[\left[X_{a-1}^{+}\left(m+n_{t}\right)\right), X_{a}^{+}(0)\right]_{q_{a}}, X_{a+1, b}^{+}(0)\right]_{q_{a+1}} v_{a, b}^{(t-1)} \\
& +\sum_{s=0}^{\left|n_{t}\right|} c_{s}\left[\left[X_{a}^{+}\left(n_{t} \mp s\right) X_{a-1}^{+}(m \pm s), X_{a+1, b}^{+}(0)\right]_{q_{a+1}} v_{a, b}^{(t-1)}\right. \\
& =q_{a}^{n_{t}} X_{a-1, b}^{+}\left(m+n_{t}\right) v_{a, b}^{(t-1)}+\sum_{s=0}^{\left|n_{t}\right|} c_{s} X_{a, b}^{+}\left(n_{t} \mp s\right) X_{a-1}^{+}(m \pm s) v_{a, b}^{(t-1)} \\
& =0 .
\end{aligned}
$$

If $i=b+1$, we consider the case with $a=M-1$ and $b=M+1$ as an example, and the proof for the general case is similar. By (A.7) and (A.8), we have

$$
\begin{aligned}
& X_{M+2}^{+}(m) v_{M-1, M+1}^{(t)}=X_{M+2}^{+}(m) X_{M-1, M+1}^{+}\left(n_{t}\right) v_{M-1, M+1}^{(t-1)} \\
& =\left[X_{M+2}^{+}(m),\left[X_{M-1, M}^{+}\left(n_{t}\right), X_{M+1}^{+}(0)\right]_{q^{-1}}\right]_{q} v_{M-1, M+1}^{(t-1)} \\
& =\left[X_{M-1, M}^{+}\left(n_{t}\right),\left[X_{M+2}^{+}(m), X_{M+1}^{+}(0)\right]_{q}\right]_{q^{-1}} v_{M-1, M+1}^{(t-1)} \\
& =q^{m}\left[X_{M-1, M}^{+}\left(n_{t}\right),\left[X_{M+2}^{+}(0), X_{M+1}^{+}(m)\right]_{q}\right]_{q^{-1}} v_{M-1, M+1}^{(t-1)} \\
& +\sum_{s=0}^{|m|} c_{S}\left[X_{M-1, M}^{+}\left(n_{t}\right), X_{M+1}^{+}( \pm s)\right]_{q^{-1}} X_{M+2}^{+}(m \mp s) v_{M-1, M+1}^{(t-1)},
\end{aligned}
$$

where the second term on the right hand side vanishes by (B.1). We can rewrite first term as $-q^{1+m}\left[\left[\left[X_{M-1}^{+}\left(n_{t}\right), X_{M}^{+}(0)\right]_{q}, X_{M+1}^{+}(m)\right]_{q^{-1}}, X_{M+2}^{+}(0)\right]_{q^{-1}} v_{M-1, M+1}^{(t-1)}$, which, by (A.1), is equal to

$$
\begin{aligned}
& -q^{1+m}\left[\left[\left[X_{M-1}^{+}\left(n_{t}\right),\left[X_{M}^{+}(0), X_{M+1}^{+}(m)\right]_{q^{-1}}\right]_{q}, X_{M+2}^{+}(0)\right]_{q^{-1}} v_{M-1, M+1}^{(t-1)}\right. \\
& =q^{m}\left[\left[\left[X_{M-1}^{+}\left(n_{t}\right),\left[X_{M+1}^{+}(m), X_{M}^{+}(0)\right]_{q}\right]_{q}, X_{M+2}^{+}(0)\right]_{q^{-1}} v_{M-1, M+1}^{(t-1)} .\right.
\end{aligned}
$$


Using A.10), we can cast the right hand side into

$$
\begin{aligned}
& q^{2 m}\left[\left[\left[X_{M-1}^{+}\left(n_{t}\right),\left[X_{M+1}^{+}(0), X_{M}^{+}(m)\right]_{q}\right]_{q}, X_{M+2}^{+}(0)\right]_{q^{-1}} v_{M-1, M+1}^{(t-1)}\right. \\
& +\sum_{k=0}^{|m|} c_{k}^{\prime}\left[\left[\left[X_{M-1}^{+}\left(n_{t}\right), X_{M}^{+}(m \pm k) X_{M+1}^{+}(\mp k)\right]_{q}, X_{M+2}^{+}(0)\right]_{q^{-1}} v_{M-1, M+1}^{(t-1)}\right.
\end{aligned}
$$

where the second term vanishes by $(\overline{B .1})$, and the first term can be rewritten as

$$
-q^{1+2 m}\left[\left[\left[X_{M-1}^{+}\left(n_{t}\right), X_{M}^{+}(m)\right]_{q}, X_{M+1}^{+}(0)\right]_{q^{-1}}, X_{M+2}^{+}(0)\right]_{q^{-1}} v_{M-1, M+1}^{(t-1)} .
$$

By (A.9), this can be expressed as

$$
\begin{aligned}
& -q^{1+3 m}\left[\left[\left[X_{M-1}^{+}\left(n_{t}+m\right), X_{M}^{+}(0)\right]_{q}, X_{M+1}^{+}(0)\right]_{q^{-1}}, X_{M+2}^{+}(0)\right]_{q^{-1}} v_{M-1, M+1}^{(t-1)} \\
& +\sum_{l=0}^{|m|} c_{l}^{\prime \prime}\left[\left[X_{M}^{+}(m \pm l) X_{M-1}^{+}\left(n_{t} \mp l\right), X_{M+1}^{+}(0)\right]_{q^{-1}}, X_{M+2}^{+}(0)\right]_{q^{-1}} v_{M-1, M+1}^{(t-1)} \\
& =-q^{1+3 m} X_{M-1, M+2}^{+}\left(n_{t}+m\right) v_{M-1, M+1}^{(t-1)}=0
\end{aligned}
$$

For $i=a(a \neq M)$, we obviously have

$$
\begin{aligned}
& X_{a}^{+}(m) v_{a, b}^{(t)}=X_{a}^{+}(m) X_{a, b}^{+}\left(n_{t}\right) v_{a, b}^{(t-1)}=\left[X_{a}^{+}(m), X_{a, b}^{+}\left(n_{t}\right)\right]_{q_{a+1}^{-1}} v_{a, b}^{(t-1)} \\
& =\left[\left[\left[X_{a}^{+}(m),\left[X_{a}^{+}\left(n_{t}\right), X_{a+1}^{+}(0)\right]_{q_{a+1}}\right]_{q_{a+1}^{-1}}, X_{a+2, b}^{+}(0)\right]_{q_{b}} v_{a, b}^{(t-1)},\right.
\end{aligned}
$$

which can be rewitten as

$$
\begin{aligned}
& q_{a}^{m-n_{t}}\left[\left[\left[X_{a}^{+}(m),\left[X_{a}^{+}(m), X_{a+1}^{+}\left(n_{t}-m\right)\right]_{q_{a+1}}\right]_{q_{a+1}^{-1}}, X_{a+2, b}^{+}(0)\right]_{q_{b}} v_{a, b}^{(t-1)}\right. \\
& +\sum_{s=0}^{\left|n_{t}-m\right|} c_{s}\left[\left[\left[X_{a}^{+}(m), X_{a+1}^{+}( \pm s) X_{a}^{+}\left(n_{t} \mp s\right)\right]_{q_{a+1}^{-1}}, X_{a+2, b}^{+}(0)\right]_{q_{b}} v_{a, b}^{(t-1)}\right.
\end{aligned}
$$

by using (A.9). The first term vanishes by (A.2), and the second terms is equal to

$$
\sum_{s=0}^{\left|n_{t}-m\right|} c_{s}\left(X_{a}^{+}(m) X_{a+1, b}^{+}( \pm s) X_{a}^{+}\left(n_{t} \mp s\right)-q_{a+1}^{-1} X_{a+1, b}^{+}( \pm s) X_{a}^{+}\left(n_{t} \mp s\right) X_{a}^{+}(m)\right) v_{a, b}^{(t-1)}
$$

which obviously vanishes. Similarly, one can prove that $X_{b}^{+}(m) v_{a, b}^{(t)}=0$. 
For $a<i<M$,

$$
\begin{aligned}
& X_{i}^{+}(m) v_{a, b}^{(t)}=\left[X_{i}^{+}(m), X_{a, b}^{+}\left(n_{t}\right)\right] v_{a, b}^{(t-1)} \\
& =\left[X_{a, i-2}^{+}\left(n_{t}\right),\left[\left[X_{i}^{+}(m),\left[X_{i-1}^{+}(0),\left[X_{i}^{+}(0), X_{i+1}^{+}(0)\right]_{q}\right]_{q}\right], X_{i+2, b}^{+}(0)\right]_{q b}\right] q v_{a, b}^{(t-1)} \\
& =q_{i}^{m}\left[X_{a, i-2}^{+}\left(n_{t}\right),\left[\left[X_{i}^{+}(m),\left[X_{i-1}^{+}(0),\left[X_{i}^{+}(m), X_{i+1}^{+}(-m)\right]_{q}\right]_{q}\right], X_{i+2, b}^{+}(0)\right]_{q_{b}}\right]_{q} v_{a, b}^{(t-1)} \\
& +\sum_{s=0}^{|m|} c_{s}\left[X_{a, i-2}^{+}\left(n_{t}\right),\left[\left[X_{i}^{+}(m),\left[X_{i-1}^{+}(0), X_{i+1}^{+}( \pm s) X_{i}^{+}(\mp s)\right]_{q}\right], X_{i+2, b}^{+}(0)\right]_{q_{b}}\right]_{q} v_{a, b}^{(t-1)}
\end{aligned}
$$

where the first term on the right hand side vanishes by lemma A.2 (1). Hence

$$
\begin{aligned}
X_{i}^{+}(m) v_{a, b}^{(t)} & =\sum_{s=0}^{|m|} c_{s}\left[X_{i}^{+}(m),\left[X_{a, i-1}^{+}\left(n_{t}\right), X_{i+1, b}^{+}( \pm s) X_{i}^{+}(\mp s)\right] q_{i}\right] v_{a, b}^{(t-1)} \\
& =0 .
\end{aligned}
$$

Similarly, one can prove that $X_{i}^{+}(m) v_{a, b}^{(t)}=0$ for $M<i<b$.

Thus we have proved that $X_{i}^{+}(m) v_{a, b}^{(t)}=0, \forall i \neq M, m \in \mathbb{Z}$.

Now we prove $X_{k, l}^{+}(m) v_{a, b}^{(t)}=0$ for all $m \in \mathbb{Z},(k, l)>(a, b)$.

For $k<a<M, l<b$,

$$
\begin{aligned}
& X_{k, l}^{+}(m) v_{a, b}^{(t)}=X_{k, l}^{+}(m) X_{a, b}^{+}\left(n_{t}\right) v_{a, b}^{(t-1)}=\left[\left[X_{k, a}^{+}(m), X_{a+1, l}^{+}(0)\right]_{q}, X_{a, b}^{+}\left(n_{t}\right)\right] v_{a, b}^{(t-1)} \\
& =\left[\left[X_{k, a}^{+}(m),\left[X_{a+1, l}^{+}(0), X_{a, b}^{+}\left(n_{t}\right)\right]\right]_{q} v_{a, b}^{(t-1)}-\left[\left[X_{k, a}^{+}(m), X_{a, b}^{+}\left(n_{t}\right)\right], X_{a+1, l}^{+}(0)\right]_{q} v_{a, b}^{(t-1)},\right.
\end{aligned}
$$

where the first term on the right hand side vanishes by Lemma A.2. It is not difficult to show that $\left[X_{k, a}^{+}(m), X_{a, b}^{+}\left(n_{t}\right)\right] v_{a, b}^{(t-1)}=0$. Hence we can rewrite the right hand side as

$$
\begin{aligned}
& -\left[X_{k, a}^{+}(m), X_{a, b}^{+}\left(n_{t}\right)\right] X_{a+1, l}^{+}(0) v_{a, b}^{(t-1)} \\
& =-\left[\left[X_{k, a-1}^{+}(m), X_{a}^{+}(0)\right]_{q},\left[X_{a}^{+}\left(n_{t}\right), X_{a+1, b}^{+}(0)\right]_{q}\right] X_{a+1, l}^{+}(0) v_{a, b}^{(t-1)} \\
& =-\left[\left[\left[X_{k, a-1}^{+}(m), X_{a}^{+}(0)\right]_{q}, X_{a}^{+}\left(n_{t}\right)\right]_{q^{-1}, X_{a+1, b}^{+}}(0)\right]_{q^{2}} X_{a+1, l}^{+}(0) v_{a, b}^{(t-1)} \\
& -q^{-1}\left[X_{a}^{+}\left(n_{t}\right),\left[\left[X_{k, a-1}^{+}(m), X_{a}^{+}(0)\right]_{q}, X_{a+1, b}^{+}(0)\right]_{q}\right]_{q^{2}} X_{a+1, l}^{+}(0) v_{a, b}^{(t-1)} \\
& =-\left[\left[\left[\left[X_{k, a-2}^{+}(m), X_{a-1}^{+}(0)\right]_{q}, X_{a}^{+}(0)\right]_{q}, X_{a}^{+}\left(n_{t}\right)\right]_{\left.q^{-1}, X_{a+1, b}^{+}(0)\right]_{q^{2}} X_{a+1, l}^{+}(0) v_{a, b}^{(t-1)}}\right. \\
& -q^{-1}\left[X_{a}^{+}\left(n_{t}\right), X_{k, b}^{+}(m)\right]_{q^{2}} X_{a+1, l}^{+}(0) v_{a, b}^{(t-1)},
\end{aligned}
$$


where the second term on the right hand side vanishes, and the first term can be rewritten as

$$
\begin{aligned}
& -\left[\left[X_{k, a-2}^{+}(m),\left[\left[X_{a-1}^{+}(0), X_{a}^{+}(0)\right]_{q}, X_{a}^{+}\left(n_{t}\right)\right]_{q^{-1}}\right]_{q}, X_{a+1, b}^{+}(0)\right]_{q^{2}} X_{a+1, l}^{+}(0) v_{a, b}^{(t-1)} \\
= & -q^{-n_{t}}\left[\left[X_{k, a-2}^{+}(m),\left[\left[X_{a-1}^{+}\left(-n_{t}\right), X_{a}^{+}\left(n_{t}\right)\right]_{q}, X_{a}^{+}\left(n_{t}\right)\right]_{q^{-1}}\right]_{q}, X_{a+1, b}^{+}(0)\right]_{q^{2}} X_{a+1, l}^{+}(0) v_{a, b}^{(t-1)} \\
& +\sum_{s=0}^{\left|n_{t}\right|} c_{s}\left[\left[X_{k, a-2}^{+}(m),\left[X_{a}^{+}( \pm s) X_{a-1}^{+}(\mp s), X_{a}^{+}\left(n_{t}\right)\right]_{q^{-1}}\right]_{q}, X_{a+1, b}^{+}(0)\right]_{q^{2}} X_{a+1, l}^{+}(0) v_{a, b}^{(t-1)}
\end{aligned}
$$

The first term on the right hand side vanishes by (A.2), and the second term can be expanded into

$$
\begin{aligned}
& \sum_{s=0}^{\left|n_{t}\right|} c_{s}\left[X_{a}^{+}( \pm s)\left[X_{k, a-2}^{+}(m), X_{a-1}^{+}(\mp s)\right]_{q}, X_{a, b}^{+}\left(n_{t}\right)\right] X_{a+1, l}^{+}(0) v_{a, b}^{(t-1)} \\
& +q^{2} \sum_{s=0}^{\left|n_{t}\right|} c_{s}\left[X_{a, b}^{+}( \pm s)\left[X_{k, a-2}^{+}(m), X_{a-1}^{+}(\mp s)\right]_{q}, X_{a}^{+}\left(n_{t}\right)\right]_{q^{-2}} X_{a+1, l}^{+}(0) v_{a, b}^{(t-1)}
\end{aligned}
$$

by using (A.1). We can show that the first term vanishes identically, and the second can be rewritten as

$$
\begin{aligned}
& q^{2} \sum_{s=0}^{\left|n_{t}\right|} c_{s} X_{a, b}^{+}( \pm s) X_{k, a-2}^{+}(m) X_{a-1}^{+}(\mp s) X_{a}^{+}\left(n_{t}\right) X_{a+1, l}^{+}(0) v_{a, b}^{(t-1)} \\
& =q^{2} \sum_{s=0}^{\left|n_{t}\right|} c_{s} X_{a, b}^{+}( \pm s) X_{k, a-2}^{+}(m) X_{a-1}^{+}(\mp s) X_{a, l}^{+}\left(n_{t}\right) v_{a, b}^{(t-1)} \\
& =q^{2} \sum_{s=0}^{\left|n_{t}\right|} c_{s} X_{a, b}^{+}( \pm s) X_{k, l}^{+}\left(m \mp s+n_{t}\right) v_{a, b}^{(t-1)} \\
& =0 .
\end{aligned}
$$

By modifying the above computations slightly, one can prove that $X_{k, l}^{+}(m) v_{a, b}^{(t)}=0$ for $k<a=M, l<b$. It is even easier to show that $X_{k, l}^{+}(m) v_{a, b}^{(t)}=0$ for $k<a, l \geq b$.

Now consider the cases $k \geq a, l>b$.

For $k<M$, we have

$$
\begin{aligned}
& X_{k, l}^{+}(m) v_{a, b}^{(t)}=X_{k, l}^{+}(m) X_{a, b}^{+}\left(n_{t}\right) v_{a, b}^{(t-1)} \\
& =\left[X_{k}^{+}(m),\left[X_{k+1, b}^{+}(0), X_{b+1, l}^{+}(0)\right]_{q^{-1}}\right]_{q_{k+1}} X_{a, b}^{+}\left(n_{t}\right) v_{a, b}^{(t-1)} \\
& =X_{k}^{+}(m)\left[X_{k+1, b}^{+}(0), X_{b+1, l}^{+}(0)\right]_{q^{-1}} X_{a, b}^{+}\left(n_{t}\right) v_{a, b}^{(t-1)} \\
& =\left(X_{k}^{+}(m) X_{k+1, b}^{+}(0) X_{b+1, l}^{+}(0)-q^{-1} X_{k}^{+}(m) X_{b+1, l}^{+}(0) X_{k+1, b}^{+}(0)\right) X_{a, b}^{+}\left(n_{t}\right) v_{a, b}^{(t-1)},
\end{aligned}
$$


which, by using Lemma A.2, can be expressed as

$$
\begin{aligned}
& -q X_{k}^{+}(m) X_{k+1, b}^{+}(0)\left(\left[X_{a, b}^{+}\left(n_{t}\right), X_{b+1, l}^{+}(0)\right]_{q^{-1}}-X_{a, b}^{+}\left(n_{t}\right) X_{b+1, l}^{+}(0)\right) v_{a, b}^{(t-1)} \\
& -q^{-2} X_{k}^{+}(m) X_{b+1, l}^{+}(0) X_{a, b}^{+}\left(n_{t}\right) X_{k+1, b}^{+}(0) v_{a, b}^{(t-1)} \\
& =-q X_{k}^{+}(m) X_{k+1, b}^{+}(0) X_{a, l}^{+}\left(n_{t}\right) v_{a, b}^{(t-1)}+q X_{k}^{+}(m) X_{k+1, b}^{+}(0) X_{a, b}^{+}\left(n_{t}\right) X_{b+1, l}^{+}(0) v_{a, b}^{(t-1)} \\
& -q^{-2} X_{k}^{+}(m) X_{b+1, l}^{+}(0) X_{a, b}^{+}\left(n_{t}\right) X_{k+1, b}^{+}(0) v_{a, b}^{(t-1)},
\end{aligned}
$$

where the first two terms on the right hand side vanishe by (B.1), and the third can be manipulated to yield

$$
\begin{aligned}
& -q^{-2} X_{k}^{+}(m)\left[X_{b+1, l}^{+}(0), X_{a, b}^{+}\left(n_{t}\right)\right]_{q} X_{k+1, b}^{+}(0) v_{a, b}^{(t-1)}+X_{k}^{+}(m) X_{a, b}^{+}\left(n_{t}\right) X_{k+1, l}^{+}(0) v_{a, b}^{(t-1)} \\
& =q^{-1} X_{k}^{+}(m) X_{a, l}^{+}(0) X_{k+1, b}^{+}(0) v_{a, b}^{(t-1)}+X_{k}^{+}(m) X_{a, b}^{+}\left(n_{t}\right) X_{k+1, l}^{+}(0) v_{a, b}^{(t-1)} \\
& =X_{k}^{+}(m) X_{a, b}^{+}\left(n_{t}\right) X_{k+1, l}^{+}(0) v_{a, b}^{(t-1)} \\
& =\left[X_{k}^{+}(m),\left[\left[X_{a, k-1}^{+}\left(n_{t}\right),\left[X_{k}^{+}(0), X_{k+1}^{+}(0)\right]_{q_{k+1}}\right]_{q_{k}}, X_{k+2, b}^{+}(0)\right]_{q_{k+2}}\right] X_{k+1, l}^{+}(0) v_{a, b}^{(t-1)} \\
& =q^{m}\left[X_{k}^{+}(m),\left[\left[X_{a, k-1}^{+}\left(n_{t}\right),\left[X_{k}^{+}(m), X_{k+1}^{+}(-m)\right]_{q_{k+1}}\right]_{q_{k}}, X_{k+2, b}^{+}(0)\right]_{q_{k+2}}\right] X_{k+1, l}^{+}(0) v_{a, b}^{(t-1)} \\
& +\sum_{s=0}^{|m|} c_{s}\left[X_{k}^{+}(m),\left[\left[X_{a, k-1}^{+}\left(n_{t}\right), X_{k+1}^{+}( \pm s) X_{k}^{+}(\mp s)\right]_{q_{k}}, X_{k+2, b}^{+}(0)\right]_{q_{k+2}}\right] X_{k+1, l}^{+}(0) v_{a, b}^{(t-1)},
\end{aligned}
$$

where the first term on the right hand side vanishes by (A.2). Hence we can rewrite the right hand side as

$$
\begin{aligned}
& \sum_{s=0}^{|m|} c_{s}\left[X_{k}^{+}(m),\left[X_{a, k-1}^{+}\left(n_{t}\right), X_{k+1, b}^{+}( \pm s) X_{k}^{+}(\mp s)\right]_{q_{k}}\right] X_{k+1, l}^{+}(0) v_{a, b}^{(t-1)} \\
& =\sum_{s=0}^{|m|} c_{s} X_{k}^{+}(m) X_{a, k-1}^{+}\left(n_{t}\right) X_{k+1, b}^{+}( \pm s) X_{k}^{+}(\mp s) X_{k+1, l}^{+}(0) v_{a, b}^{(t-1)} \\
& =\sum_{s=0}^{|m|} c_{s} X_{k}^{+}(m) X_{a, k-1}^{+}\left(n_{t}\right) X_{k+1, b}^{+}( \pm s) X_{k, l}^{+}(\mp s) v_{a, b}^{(t-1)} \\
& =0 .
\end{aligned}
$$

Now we consider the case $k=a=M, l>b$. Since

$$
X_{M, l}^{+}(m) v_{M, b}^{(t)}=\left[X_{M, b+1}^{+}(m), X_{b+2, l}^{+}(0)\right]_{q^{-1}} v_{M, b}^{(t)}=-q^{-1} X_{b+2, l}^{+}(0) X_{M, b+1}^{+}(m) v_{M, b}^{(t)},
$$

it is sufficient to show that $X_{M, b+1}^{+}(m) v_{M, b}^{(t)}=0$.

By (A.10) and $X_{i}^{+}(m) v_{M, b}^{(t-1)}=X_{i}^{+}(m) v_{M, b}^{(t)}=0, i \neq M$, we have

$$
X_{M, b+1}^{+}(m) X_{M, b}^{+}\left(n_{t}\right) v_{M, b}^{(t-1)}=\left[X_{M, b}^{+}(0), X_{b+1}^{+}(m)\right]_{q^{-1}}\left[X_{M, b-1}^{+}(0), X_{b}^{+}\left(n_{t}\right)\right]_{q^{-1}} v_{M, b}^{(t-1)} .
$$


Hence,

$$
\begin{aligned}
& X_{M, b+1}^{+}(m) v_{M, b}^{(t)}=X_{M, b+1}^{+}(m) X_{M, b}^{+}\left(n_{t}\right) v_{M, b}^{(t-1)} \\
& =\left[\left[X_{M, b}^{+}(0), X_{b+1}^{+}(m)\right]_{q^{-1}},\left[X_{M, b-1}^{+}(0), X_{b}^{+}\left(n_{t}\right)\right]_{q^{-1}}\right]_{q} v_{M, b}^{(t-1)} \\
& =\left[\left[\left[X_{M, b}^{+}(0), X_{b+1}^{+}(m)\right]_{q^{-1}}, X_{M, b-1}^{+}(0)\right]_{q}, X_{b}^{+}\left(n_{t}\right)\right]_{q^{-1}} v_{M, b}^{(t-1)} \\
& -q\left[X_{M, b-1}^{+}(0),\left[\left[X_{M, b}^{+}(0), X_{b+1}^{+}(m)\right]_{q^{-1}}, X_{b}^{+}\left(n_{t}\right)\right]\right]_{q^{-2} v_{M, b}^{(t-1)}} \\
& =-q\left[X_{M, b-1}^{+}(0),\left[\left[X_{M, b-2}^{+}(0),\left[X_{b-1}^{+}(0),\left[X_{b}^{+}(0), X_{b+1}^{+}(m)\right]_{q^{-1}}\right]_{q^{-1}}\right]_{q^{-1}}, X_{b}^{+}\left(n_{t}\right)\right]\right]_{q^{-2}} v_{M, b}^{(t-1)} \\
& =\left[X_{M, b-1}^{+}(0),\left[X_{M, b-2}^{+}(0),\left[\left[X_{b-1}^{+}(0),\left[X_{b+1}^{+}(m), X_{b}^{+}(0)\right]_{q}\right]_{q^{-1}}, X_{b}^{+}\left(n_{t}\right)\right]\right]_{q^{-1}}\right]_{q^{-2}} v_{M, b}^{(t-1)} \\
& =q^{n_{t}}\left[X_{M, b-1}^{+}(0),\left[X_{M, b-2}^{+}(0),\left[\left[X_{b-1}^{+}(0),\left[X_{b+1}^{+}\left(m-n_{t}\right), X_{b}^{+}\left(n_{t}\right)\right]_{q}\right]_{q^{-1}}, X_{b}^{+}\left(n_{t}\right)\right]\right]_{q^{-1}}\right]_{q^{-2}} v_{M, b}^{(t-1)} \\
& +\sum_{s=0}^{\left|n_{t}\right|} c_{s}\left[X_{M, b-1}^{+}(0),\left[X_{M, b-2}^{+}(0),\left[\left[X_{b-1}^{+}(0), X_{b}^{+}( \pm s) X_{b+1}^{+}(m \mp s)\right]_{q^{-1}}, X_{b}^{+}\left(n_{t}\right)\right]\right]_{q^{-1}}\right]_{q^{-2}} v_{M, b}^{(t-1)} \\
& =\sum_{s=0}^{\left|n_{t}\right|} c_{S}\left[X_{M, b-1}^{+}(0),\left[\left[X_{M, b-1}^{+}(0), X_{b}^{+}( \pm s) X_{b+1}^{+}(m \mp s)\right]_{q^{-1}}, X_{b}^{+}\left(n_{t}\right)\right]\right]_{q^{-2}} v_{M, b}^{(t-1)} \\
& =\sum_{s=0}^{\left|n_{t}\right|} c_{s}\left[X_{M, b-1}^{+}(0),\left[\left[X_{M, b-1}^{+}(0), X_{b}^{+}( \pm s)\right]_{q^{-1}} X_{b+1}^{+}(m \mp s), X_{b}^{+}\left(n_{t}\right)\right]\right]_{q^{-2} v_{M, b}^{(t-1)}} \\
& =\sum_{s=0}^{\left|n_{t}\right|} c_{s}\left[\left[X_{M, b-1}^{+}(0), X_{b}^{+}( \pm s)\right]_{q^{-1}} X_{b+1}^{+}(m \mp s), X_{b}^{+}\left(n_{t}\right)\right] X_{M, b-1}^{+}(0) v_{M, b}^{(t-1)} \\
& =\sum_{s=0}^{\left|n_{t}\right|} c_{s}\left[X_{M, b-1}^{+}(0), X_{b}^{+}( \pm s)\right]_{q^{-1}} X_{b+1}^{+}(m \mp s) X_{b}^{+}\left(n_{t}\right) X_{M, b-1}^{+}(0) v_{M, b}^{(t-1)} \\
& +X_{b}^{+}\left(n_{t}\right)\left[X_{M, b-1}^{+}(0), X_{b}^{+}( \pm s)\right]_{q^{-1}} X_{b+1}^{+}(m \mp s) X_{M, b-1}^{+}(0) v_{M, b}^{(t-1)} \\
& =\sum_{s=0}^{\left|n_{t}\right|} c_{s}\left[X_{M, b-1}^{+}(0), X_{b}^{+}( \pm s)\right]_{q^{-1}} X_{b+1}^{+}(m \mp s) X_{b}^{+}\left(n_{t}\right) X_{M, b-1}^{+}(0) v_{M, b}^{(t-1)} \\
& =\sum_{s=0}^{\left|n_{t}\right|} c_{s}\left[X_{M, b-1}^{+}(0), X_{b}^{+}( \pm s)\right]_{q^{-1}} X_{M, b+1}^{+}\left(m \mp s+n_{t}\right) v_{M, b}^{(t-1)} \\
& =0 \text {. }
\end{aligned}
$$

This completes the proof of the Lemma.

\section{Appendix C. Proof of Lemma 3.7}

Proof of Lemma 3.7 Note that (3.13) directly follows from (3.12). Hence we only need to prove (3.12). 
We first show that

$$
X_{a, b}^{+}(p) X_{a, b}^{+}(k) X_{a, b}^{+}(l) v_{a, b}=0 \quad \text { for all } p, k, l \in \mathbb{Z} \text { with } p \equiv l(\bmod 2) .
$$

For $a<M$, we have

$$
\begin{aligned}
& {\left[X_{a, b}^{+}(p), X_{a, b}^{+}(p+1)\right]} \\
& =\left[\left[X_{a}^{+}(p), X_{a+1, b}^{+}(0)\right]_{q},\left[X_{a}^{+}(p+1), X_{a+1, b}^{+}(0)\right]_{q}\right] \\
& \left.=\left[\left[X_{a}^{+}(p), X_{a+1, b}^{+}(0)\right]_{q}, X_{a}^{+}(p+1)\right]_{q^{-1}}, X_{a+1, b}^{+}(0)\right]_{q^{2}} \\
& +q^{-1}\left[X_{a}^{+}(p+1),\left[\left[X_{a, b}^{+}(p), X_{a+1, b}^{+}(0)\right]_{q}\right]_{q^{2}}\right. \\
& \left.=\left[\left[\left[X_{a}^{+}(p), X_{a+1}^{+}(0)\right]_{q}, X_{a}^{+}(p+1)\right]_{q^{-1}}, X_{a+2, b}^{+}(0)\right]_{q_{a+2}}, X_{a+1, b}^{+}(0)\right] \\
& \left.=-q\left[\left[\left[X_{a+1}^{+}(0), X_{a}^{+}(p)\right]_{q^{-1}}, X_{a}^{+}(p+1)\right]_{q^{-1}}, X_{a+2, b}^{+}(0)\right]_{q_{a+2}}, X_{a+1, b}^{+}(0)\right] \\
& \left.=q\left[\left[\left[X_{a}^{+}(p+1), X_{a+1}^{+}(-1)\right]_{q^{-1}}, X_{a}^{+}(p+1)\right]_{q^{-1}}, X_{a+2, b}^{+}(0)\right]_{q_{a+2}}, X_{a+1, b}^{+}(0)\right] \\
& =0 \text {. }
\end{aligned}
$$

Similarly, one can show that $\left[X_{M, b}^{+}(p), X_{M, b}^{+}(p+1)\right]=0, b>M$.

From (3.11), we have

$$
X_{a, b}^{+}(p) X_{a, b}^{+}(p \pm 1) X_{a, b}^{+}(l) v_{a, b}=0 \quad \text { for all } p \equiv l(\bmod 2) .
$$

This establishes (C.1) for $|p-k|=1$.

We now use induction on $|p-k|$ to prove ([C.1). By the induction hypothesis, for all $p, k, l \in \mathbb{Z}$ with $p \equiv l(\bmod 2),|p-k| \leq 2 i-1$,

$$
X_{a, b}^{+}(p) X_{a, b}^{+}(k) X_{a, b}^{+}(l) v_{a, b}=0 .
$$

We now consider $X_{a, b}^{+}(p) X_{a, b}^{+}(p+1+2 i) X_{a, b}^{+}(l) v_{a, b}$.

For $a<M$, we have

$$
\begin{aligned}
& {\left[X_{a, b}^{+}(p), X_{a, b}^{+}(p+1+2 i)\right]} \\
& =\left[X_{a, b}^{+}(p),\left[X_{a}^{+}(p+1+2 i), X_{a+1, b}^{+}(0)\right]_{q}\right] \\
& =\left[\left[X_{a, b}^{+}(p), X_{a}^{+}(p+1+2 i)\right]_{q^{-1}, X_{a+1, b}^{+}}(0)\right]_{q^{2}} \\
& +q^{-1}\left[X_{a}^{+}(p+1+2 i),\left[X_{a, b}^{+}(p), X_{a+1, b}^{+}(0)\right]_{q}\right]_{q^{2}}
\end{aligned}
$$

where the second term on the right hand side vanishes by Lemma A.2, and the first can be rewritten as

$$
\begin{aligned}
& {\left[\left[\left[\left[X_{a}^{+}(p), X_{a+1}^{+}(0)\right]_{q}, X_{a}^{+}(p+1+2 i)\right]_{q^{-1}}, X_{a+2, b}^{+}(0)\right]_{q_{a+2}}, X_{a+1, b}^{+}(0)\right]_{q^{2}}} \\
& =q^{2 i}\left[\left[\left[\left[X_{a}^{+}(p+2 i), X_{a+1}^{+}(-2 i)\right]_{q}, X_{a}^{+}(p+1+2 i)\right]_{\left.\left.q^{-1}, X_{a+2, b}^{+}(0)\right]_{q_{a+2}}, X_{a+1, b}^{+}(0)\right]_{q^{2}}}\right.\right. \\
& +\sum_{s=1}^{2 i} c_{s}\left[\left[\left[X_{a+1}^{+}(-s) X_{a}^{+}(p+s), X_{a}^{+}(p+1+2 i)\right]_{q^{-1}}, X_{a+2, b}^{+}(0)\right]_{q_{a+2}}, X_{a+1, b}^{+}(0)\right]_{q^{2}}
\end{aligned}
$$


We note that the first term on the right hand side vanishes:

$$
\begin{aligned}
& \left.\left[\left[X_{a}^{+}(p+2 i), X_{a+1}^{+}(-2 i)\right]_{q}, X_{a}^{+}(p+1+2 i)\right]_{q^{-1}}, X_{a+2, b}^{+}(0)\right]_{q_{a+2}} \\
& \left.=-q\left[\left[X_{a+1}^{+}(-2 i), X_{a}^{+}(p+2 i)\right]_{q^{-1}, X_{a}^{+}}(p+1+2 i)\right]_{q^{-1}}, X_{a+2, b}^{+}(0)\right]_{q_{a+2}} \\
& \left.=q\left[\left[X_{a}^{+}(p+2 i+1), X_{a+1}^{+}(-2 i-1)\right]_{q^{-1}}, X_{a}^{+}(p+1+2 i)\right]_{q^{-1}}, X_{a+2, b}^{+}(0)\right]_{q_{a+2}} \\
& =0 .
\end{aligned}
$$

Hence

$$
\begin{aligned}
& {\left[X_{a, b}^{+}(p), X_{a, b}^{+}(p+1+2 i)\right]} \\
& =\sum_{s=1}^{2 i} c_{s}\left[\left[\left[X_{a+1}^{+}(-s) X_{a}^{+}(p+s), X_{a}^{+}(p+1+2 i)\right]_{q^{-1}}, X_{a+2, b}^{+}(0)\right]_{q_{a+2}}, X_{a+1, b}^{+}(0)\right]_{q^{2}} \\
& =\sum_{s=1}^{2 i} c_{s}\left[\left[X_{a+1, b}^{+}(-s) X_{a}^{+}(p+s), X_{a}^{+}(p+1+2 i)\right]_{\left.q^{-1}, X_{a+1, b}^{+}(0)\right]_{q^{2}} .}\right.
\end{aligned}
$$

By (3.11), $X_{a, b}^{+}(p) X_{a, b}^{+}(p+1+2 i) X_{a, b}^{+}(l) v_{a, b}=\left[X_{a, b}^{+}(p), X_{a, b}^{+}(p+1+2 i)\right] X_{a, b}^{+}(l) v_{a, b}$. Hence

$$
\begin{aligned}
& X_{a, b}^{+}(p) X_{a, b}^{+}(p+1+2 i) X_{a, b}^{+}(l) v_{a, b} \\
& =\sum_{s=1}^{2 i} c_{s}\left[\left[X_{a+1, b}^{+}(-s) X_{a}^{+}(p+s), X_{a}^{+}(p+1+2 i)\right]_{q^{-1}}, X_{a+1, b}^{+}(0)\right]_{q^{2}} X_{a, b}^{+}(l) v_{a, b} .
\end{aligned}
$$

We can rewrite the right hand side as

$$
\begin{aligned}
& \sum_{s=1}^{2 i} c_{s}\left(X_{a+1, b}^{+}(-s) X_{a}^{+}(p+s) X_{a}^{+}(p+1+2 i)\right. \\
& \left.-q^{-1} X_{a}^{+}(p+1+2 i) X_{a+1, b}^{+}(-s) X_{a}^{+}(p+s)\right) X_{a+1, b}^{+}(0) X_{a, b}^{+}(l) v_{a, b} \\
& =\sum_{s=1}^{2 i} c_{s} X_{a+1, b}^{+}(-s) X_{a}^{+}(p+s) X_{a, b}^{+}(p+1+2 i) X_{a, b}^{+}(l) v_{a, b} \\
& -\sum_{s=1}^{2 i} q^{-1} c_{s} X_{a}^{+}(p+1+2 i) X_{a+1, b}^{+}(-s) X_{a, b}^{+}(p+s) X_{a, b}^{+}(l) v_{a, b} .
\end{aligned}
$$


Note that the first term on the right hand side vanishes by (3.11), and by using (3.11) and A.9 we can rewrite the second term as

$$
\begin{aligned}
& -\sum_{s=1}^{2 i} q^{-1} c_{s}\left[\left[X_{a}^{+}(p+1+2 i), X_{a+1}^{+}(-s)\right]_{q}, X_{a+2, b}^{+}(0)\right]_{q_{a+2}} X_{a, b}^{+}(p+s) X_{a, b}^{+}(l) v_{a, b} \\
& =\sum_{s=1}^{2 i} d_{s}\left[\left[X_{a}^{+}(p+1+2 i-s), X_{a+1}^{+}(0)\right]_{q}, X_{a+2, b}^{+}(0)\right]_{q_{a+2}} X_{a, b}^{+}(p+s) X_{a, b}^{+}(l) v_{a, b} \\
& +\sum_{s=1}^{2 i} d_{s}^{\prime} \sum_{r=0}^{s-1} c_{r}\left[X_{a+1}^{+}(-s+r) X_{a}^{+}(p+1+2 i-r), X_{a+2, b}^{+}(0)\right]_{q_{a+2}} X_{a, b}^{+}(p+s) X_{a, b}^{+}(l) v_{a, b} .
\end{aligned}
$$

The second term on the right hand side varnishes by (3.11). This leads to

$$
\begin{aligned}
& X_{a, b}^{+}(p) X_{a, b}^{+}(p+1+2 i) X_{a, b}^{+}(l) v_{a, b} \\
& =\sum_{s=1}^{2 i} d_{s} X_{a, b}^{+}(p+1+2 i-s) X_{a, b}^{+}(p+s) X_{a, b}^{+}(l) v_{a, b} .
\end{aligned}
$$

We observe that $|(p+1+2 i-s)-(p+s)|=|1+2 i-2 s| \leq 2 i$ for $1 \leq s \leq 2 i-1$. Thus $X_{a, b}^{+}(p) X_{a, b}^{+}(p+1+2 i) X_{a, b}^{+}(l) v_{a, b}=0$ by (‥2).

For $a=M$, by using (A.6), we obtain

$$
\begin{aligned}
& {\left[X_{M, b}^{+}(p), X_{M, b}^{+}(p+1+2 i)\right]=\left[X_{M, b}^{+}(p),\left[X_{M}^{+}(p+1+2 i), X_{M+1, b}^{+}(0)\right]_{q^{-1}}\right]} \\
& =\left[\left[X_{M, b}^{+}(p), X_{M}^{+}(p+1+2 i)\right]_{q^{-1}}, X_{M+1, b}^{+}(0)\right] \\
& -q^{-1}\left[X_{M}^{+}(p+1+2 i),\left[X_{M, b}^{+}(p), X_{M+1, b}^{+}(0)\right]_{q}\right]
\end{aligned}
$$

where the second term on the right hand side varnishes by Lemma A.2. We note that

$$
\begin{aligned}
& {\left[X_{M, b}^{+}(p), X_{M}^{+}(p+1+2 i)\right]_{q^{-1}}} \\
& =\left[\left[\left[X_{M}^{+}(p), X_{M+1}^{+}(0)\right]_{q^{-1}}, X_{M}^{+}(p+1+2 i)\right]_{q^{-1}, X_{M+2, b}^{+}}(0)\right]_{q^{-1}} \\
& =q^{-1}\left[\left[\left[X_{M}^{+}(p+1), X_{M+1}^{+}(-1)\right]_{q}, X_{M}^{+}(p+1+2 i)\right]_{q^{-1}}, X_{M+2, b}^{+}(0)\right]_{q^{-1}} \\
& =q^{2 i-1}\left[\left[\left[X_{M}^{+}(p+1+2 i), X_{M+1}^{+}(-1-2 i)\right]_{q}, X_{M}^{+}(p+1+2 i)\right]_{q^{-1}}, X_{M+2, b}^{+}(0)\right]_{q^{-1}} \\
& +\sum_{s=0}^{2 i-1} c_{s}\left[\left[X_{M}^{+}(p+1+s) X_{M+1}^{+}(-1-s), X_{M}^{+}(p+1+2 i)\right]_{q^{-1}}, X_{M+2, b}^{+}(0)\right]_{q^{-1}} \\
& =\sum_{s=0}^{2 i-1} c_{S}\left[X_{M}^{+}(p+1+s) X_{M+1, b}^{+}(-1-s), X_{M}^{+}(p+1+2 i)\right]_{q^{-1}} .
\end{aligned}
$$


Hence

$$
\begin{aligned}
& {\left[X_{M, b}^{+}(p), X_{M, b}^{+}(p+1+2 i)\right]} \\
& =\sum_{s=0}^{2 i-1} c_{S}\left[\left[X_{M}^{+}(p+1+s) X_{M+1, b}^{+}(-1-s), X_{M}^{+}(p+1+2 i)\right]_{q^{-1}}, X_{M+1, b}^{+}(0)\right] .
\end{aligned}
$$

Now we have

$$
\begin{aligned}
& X_{M, b}^{+}(p) X_{M, b}^{+}(p+1+2 i) X_{M, b}^{+}(l) v_{M, b} \\
&= {\left[X_{M, b}^{+}(p), X_{M, b}^{+}(p+1+2 i)\right] X_{M, b}^{+}(l) v_{M, b} \quad \text { by } \underline{\text { C. } 2)} } \\
&= \sum_{s=0}^{2 i-1} c_{s}\left[\left[X_{M}^{+}(p+1+s) X_{M+1, b}^{+}(-1-s), X_{M}^{+}(p+1+2 i)\right]_{\left.q^{-1}, X_{M+1, b}^{+}(0)\right] X_{M, b}^{+}(l) v_{M, b}}\right. \\
&= \sum_{s=0}^{2 i-1} c_{s} X_{M+1, b}^{+}(0) X_{M}^{+}(p+1+s) X_{M+1, b}^{+}(-1-s) X_{M}^{+}(p+1+2 i) X_{M, b}^{+}(l) v_{M, b} \quad \text { by (3.11) } \\
&= \sum_{s=0}^{2 i-1} c_{s} X_{M+1, b}^{+}(0) X_{M}^{+}(p+1+s)\left[X_{M+1, b}^{+}(-1-s), X_{M}^{+}(p+1+2 i)\right]_{q} X_{M, b}^{+}(l) v_{M, b}
\end{aligned}
$$

By (A.7) and (3.11), we note that,

$$
\begin{aligned}
& {\left[X_{M+1, b}^{+}(-1-s), X_{M}^{+}(p+1+2 i)\right]_{q} X_{M, b}^{+}(l) v_{M, b}} \\
& =\left[\left[X_{M+1}^{+}(-1-s), X_{M}^{+}(p+1+2 i)\right]_{q}, X_{M+2, b}^{+}(0)\right]_{q^{-1}} X_{M, b}^{+}(l) v_{M, b} \\
& =\sum_{s=0}^{2 i-1} q^{-1-s}\left[\left[X_{M+1}^{+}(0), X_{M}^{+}(p+2 i-s)\right]_{q}, X_{M+2, b}^{+}(0)\right]_{q^{-1}} X_{M, b}^{+}(l) v_{M, b} \\
& +\sum_{s=0}^{2 i-1} \sum_{r=0}^{s} c_{r}\left[X_{M}^{+}(p+1+2 i-r) X_{M+1}^{+}(r-1-s), X_{M+2, b}^{+}(0)\right]_{q^{-1}} X_{M, b}^{+}(l) v_{M, b} \\
& =\sum_{s=0}^{2 i-1} q^{-1-s}\left[\left[X_{M+1}^{+}(0), X_{M}^{+}(p+2 i-s)\right]_{q}, X_{M+2, b}^{+}(0)\right]_{q^{-1}} X_{M, b}^{+}(l) v_{M, b} \quad \text { by (3.11) } \\
& =\sum_{s=0}^{2 i-1} q^{-s} X_{M, b}^{+}(p+2 i-s) X_{M, b}^{+}(l) v_{M, b} .
\end{aligned}
$$


Hence,

$$
\begin{aligned}
& X_{M, b}^{+}(p) X_{M, b}^{+}(p+1+2 i) X_{M, b}^{+}(l) v_{M, b} \\
& =\sum_{s=0}^{2 i-1} c_{s} q^{-s} X_{M+1, b}^{+}(0) X_{M}^{+}(p+1+s) X_{M, b}^{+}(p+2 i-s) X_{M, b}^{+}(l) v_{M, b} \\
& =\sum_{s=0}^{2 i-1} c_{s} q^{-s}\left[X_{M+1, b}^{+}(0) X_{M}^{+}(p+1+s)\right]_{q} X_{M, b}^{+}(p+2 i-s) X_{M, b}^{+}(l) v_{M, b} \quad \text { by (3.11) } \\
& =-\sum_{s=0}^{2 i-1} c_{s} q^{1-s} X_{M, b}^{+}(p+1+s) X_{M, b}^{+}(p+2 i-s) X_{M, b}^{+}(l) v_{M, b} .
\end{aligned}
$$

We observe that $X_{M, b}^{+}(p+2 i) X_{M, b}^{+}(l) v_{M, b}=0$ by (3.11) since $p+2 i \equiv l(\bmod 2)$, and $|p+2 i-s-(p+1+s)| \leq 2 i-1$ for $1 \leq s \leq 2 i-1$. Hence, from (C.2) and the above equality we have $X_{M, b}^{+}(p) X_{M, b}^{+}(p+1+2 i) X_{M, b}^{+}(l) v_{M, b}=0$. Now we have prove that

$$
X_{a, b}^{+}(p) X_{a, b}^{+}(p+1+2 i) X_{a, b}^{+}(l) v_{a, b}=0 \quad(a, b) \in S .
$$

Similarly, one can prove that

$$
X_{a, b}^{+}(p) X_{a, b}^{+}(p-1-2 i) X_{a, b}^{+}(l) v_{a, b}=0 .
$$

This completes the proof of (C.1).

Using the similar arguments in the proof of (C.1) one can prove that

$$
X_{a, b}^{+}(p) X_{a, b}^{+}(k) X_{a, b}^{+}(l) v_{a, b}=0 \quad \text { for all } p, k, l \in \mathbb{Z} \text { with } p \equiv k(\bmod 2) .
$$

Now (3.12) follows from (C.1), (C.5) and (3.11).

Acknowledgement. This work was supported by the Chinese National Natural Science Foundation grant No. 11271056, Australian Research Council Discovery-Project Grants DP0986349 and DP140103239, Qing Lan Project of Jiangsu Province, and Jiangsu Overseas Research and Training Program for Prominent Young and Middle Aged University Teachers and Presidents. Part of this work was completed when both authors visited the University of Science and Technology of China.

\section{REFERENCES}

[1] G. Benkart, S-J. Kang, M. Kashiwara, Crystal bases for the quantum superalgebra $\mathrm{U}_{q}(\mathfrak{g l}(m, n))$. J. Amer. Math. Soc. 13 (2000) 295-331.

[2] A. J. Bracken, M. D. Gould and R. B. Zhang, Quantum supergroups and solutions of the YangBaxter Equation. Modern Physics Letters A5 (1990) no. 11, 831-840.

[3] J. Beck, V. Chari, A. Pressley, An algebraic characterization of the affine canonical basis. Duke Math. J. 99 (1999), no.3, 455-487.

[4] M. Chaichian and P. Kulish, Quantum Lie superalgebras and q-oscillators. Phys. Lett. B 234 (1990) 72-80. 
[5] V. Chari, Integral representations of affine Lie algebra. Invent. Math. 85 (1986), 317-335.

[6] V. Chari and J. Greenstein, Quantun loop modules. Represent. Theory 7 (2003), 56-80.

[7] V. Chari and A. Pressley, New unitary representations of loop groups, Math. Ann. 275 (1986), $87-104$.

[8] V. Chari and A. Pressley, Quantum affine algebras. Commun. Math. Phys. 142 (1991), 261-283.

[9] V. Chari and A. Pressley, Weyl modules for classical and quantum affine algebras. Represent. Theory 5 (2001), 191-223.

[10] A. Foerster and M. Karowski, The supersymmetric t-J model with quantum group invariance. Nuclear Phys. B 408 (1993), no. 3, 512-534.

[11] R. Floreanini, D. A. Leites and L. Vinet, On the defining relations of quantum superalgebras. Lett. Math. Phys. 23 (1991), no. 2, 127-131.

[12] P. D. Jarvis and R. B. Zhang, Affine superalgebras, unitary representations, and the Sugawara construction, Nucl. Phys. B313 (1989), 205-219.

[13] J. R. Links, M. D. Gould and R. B. Zhang, Quantum supergroups, link polynomials and representation of the braid generator. Rev. Math. Phys. 5 (1993), no. 2, 345-361.

[14] V. Kac, Lie superalgebras, Adv. Math. 26 (1977), 8-96.

[15] V. Kac and M. Wakimoto, Integrable highest weight modules over affine superalgebras and Appell's function, Comm. Math. Phys. 215 (2001), 631-682.

[16] S. M. Khoroshkin and V. N. Tolstoy, Universal $R$-matrix for quantized (super)algebras. Commun. in Math. Physics 141 (1991), no. 3, 599-617.

[17] E. Lanzmann, The Zhang transformation and $\mathrm{U}_{q}(\operatorname{osp}(1,2 \ell))$-Verma modules annihilators. Algebras and Rep. Theory 5 (2002), no. 3, 235-258.

[18] G. Lusztig, Introduction to quantum groups. Progress in Mathematics, 110. Birkhuser Boston, Inc., Boston, MA, 1993.

[19] I. M. Musson and Y. M. Zou, Crystal bases for $\mathrm{U}_{q}(\operatorname{osp}(1,2 r))$. J. Algebra 210 (1998), no. 2, 514-534.

[20] T. D. Palev and N. I. Stoilova, Highest weight irreducible representations of the quantum algebra $\mathrm{U}_{h}\left(A_{\infty}\right)$. J. Math. Phys. 39 (1998), no. 11, 5832-5849.

[21] T. D. Palev, N. I. Stoilova, J. Van der Jeugt, Finite-dimensional representations of the quantum superalgebra $\mathrm{U}_{q}(\mathfrak{g l}(n \mid m))$ and related $q$-identities. Commun. Math. Phys. 166 (1994), no. 2, 367-378.

[22] S. Rao, Iterated loop modules and a filtration for vertex representations of toroidal Lie algebras. Pacific J. Math. 171(2) (1995), 511-528.

[23] S. Rao, Classification of irreducible integrable modules for multi loop algebras with finite dimensional weight spaces, J. Algebra 246 (2001), 215-225.

[24] S. Rao, Complete reducibility of integrable modules for the affine Lie (super)algebras, J. Algebra 264 (2003) 269-278.

[25] S. Rao, Finite dimensional modules for multiloop superalgebra of type $A(m, n)$ and $C(m)$. Proc. Amer. Math. Soc. 141 (2013) 3411-3419.

[26] S. Rao and K. Zhao, On integrable representations for toroidal Lie superalgebras, Contemp. Math. 343 (2004), 243-261.

[27] M. Scheunert, Serre-type relations for special linear Lie superalgebras. Lett. Math. Phys. 24 (1992), no. 3, 173-181.

[28] Yuezhu Wu and R. B. Zhang, Unitary highest weight representations of quantum general linear superalgebra. J. Algebra 321 (2009), no. 11, 3568-3593.

[29] Yuezhu Wu and R. B. Zhang, Integrable representations of affine $A(m, n)$ and $C(m)$ superalgebras, arXiv:1309.1533 
[30] H. Yamane, Quantized enveloping algebras associated with simple Lie superalgebras and their universal $R$ - matrices. Publ. RIMS. Kyoto Univ. 30 (1994) 15-87.

[31] H. Yamane, On definding relations of the affine Lie superalgebras and their quantized universal enveloping superalgebras. Publ. RIMS. Kyoto Univ. 35 (1999) 321-390.

[32] W.-L. Yang and Y.-Z. Zhang, Highest weight representations of $\mathrm{U}_{q}(\widehat{\operatorname{sl}}(2 \mid 1))$ and correlation functions of the q-deformed supersymmetric t-J model. Nuclear Phys. B 547 (1999), no. 3, 599-622.

[33] Hechun Zhang, The quantum general linear supergroup, canonical bases and Kazhdan-Lusztig polynomials. Science in China. Series A, Mathematics 52, (2009), no.3, 401-416.

[34] Huafeng Zhang, Representations of quantum affine superalgebras. arXiv:1309.5250

[35] R. B. Zhang, Universal $\mathrm{L}$ operator and invariants of the quantum supergroup $\mathrm{U}_{q}(g l(m / n)) . J$. Math. Phys. 33 (1992), no. 6, 1970 - 1979.

[36] R. B. Zhang, Braid group representations arising from quantum supergroups with arbitrary q and link polynomials. J. Math. Phys. 33 (1992), no. 11, 3918-3930.

[37] R. B. Zhang, Finite-dimensional representations of $\mathrm{U}_{q}(\operatorname{osp}(1 / 2 n))$ and its connection with quantum so $(2 n+1)$. Lett. Math. Phys. 25 (1992), no. 4, 317-325.

[38] R. B. Zhang, Finite-dimensional irreducible representations of the quantum supergroup $\mathrm{U}_{q}(g l(m / n))$. J. Math. Phys. 34 (1993), no. 3, 1236-1254.

[39] R. B. Zhang, Quantum supergroups and topological invariants of three-manifolds. Rev. Math. Phys. 7 (1995), no. 5, 809-831.

[40] R. B. Zhang, The $\mathfrak{g l}(M \mid N)$ super Yangian and its finite dimensional representations. Lett. Math. Phys. 37 (1996) 419-434.

[41] R. B. Zhang, Symmetrizable quantum affine superalgebras and their representations. J. Math. Phys. 38 (1997) 535-543.

[42] R. B. Zhang, Structure and representations of the quantum general linear supergroup. Comm. Math. Phys. 195 (1998), no. 3, 525-547.

[43] R. B. Zhang, Quantum superalgebra representations on cohomology groups of non-commutative bundles. J. Pure Appl. Algebra 191 (2004), no. 3, 285-314.

[44] R. B. Zhang, A. J. Bracken and M. D. Gould, Solution of the graded Yang-Baxter equation associated with the vector representation of $\mathrm{U}_{q}(\operatorname{osp}(M / 2 n))$. Phys. Lett. B 257 (1991), no. 1-2, 133-139.

[45] R. B. Zhang, M. D. Gould and A. J. Bracken, Solutions of the graded classical Yang-Baxter equation and integrable models. J. Phys. A 24 (1991), no. 6, 1185-1197.

[46] Y. M. Zou, Integrable representations of $\mathrm{U}_{q}(\operatorname{osp}(1,2 n))$. J. Pure Appl. Algebra 130 (1998), no. $1,99-112$.

[47] Y. M. Zou, Crystal bases for $\mathrm{U}_{q}\left(\Gamma\left(\sigma_{1}, \sigma_{2}, \sigma_{3}\right)\right)$. Trans. Amer. Math. Soc. 353 (2001), no. 9 , 3789-3802.

(Wu) School of Mathematics and Statistics, Changshu Institute of Technology, Changshu, Jiangsu, China

(Wu, Zhang) School of Mathematics and Statistics, University of Sydney, Sydney, NSW 2006, Australia

E-mail address: yuezhuwu@maths . usyd.edu. au

E-mail address: ruibin.zhang@sydney.edu.au 